\title{
Partially composite Higgs models: phenomenology and RG analysis
}

\author{
Tommi Alanne, ${ }^{a}$ Diogo Buarque Franzosi, ${ }^{b}$ Mads T. Frandsen, ${ }^{c}$ \\ Mette L.A. Kristensen, ${ }^{c}$ Aurora Meroni ${ }^{d}$ and Martin Rosenlyst ${ }^{c}$ \\ ${ }^{a}$ Max-Planck-Institut für Kernphysik, \\ Saupfercheckweg 1, 69117 Heidelberg, Germany \\ ${ }^{b}$ Institut für Theoretische Physik, Universität Gättingen, \\ Friedrich-Hund-Platz 1, 37077 Göttingen, Germany \\ ${ }^{c} C P^{3}$-Origins, University of Southern Denmark, \\ Campusvej 55, DK-5230 Odense M, Denmark \\ ${ }^{d}$ Department of Physics, University of Helsinki $\mathscr{G}$ Helsinki Institute of Physics, \\ P.O.Box 64, FI-00014 University of Helsinki, Finland \\ E-mail: tommi.alanne@mpi-hd.mpg.de, dbuarqu@gwdg.de, \\ frandsen@cp3.sdu.dk, metkr03@student.sdu.dk, \\ aurora.meroni@helsinki.fi, rosenlyst@cp3.sdu.dk
}

ABSTRACT: We study the phenomenology of partially composite-Higgs models where electroweak symmetry breaking is dynamically induced, and the Higgs is a mixture of a composite and an elementary state. The models considered have explicit realizations in terms of gauge-Yukawa theories with new strongly interacting fermions coupled to elementary scalars and allow for a very SM-like Higgs state. We study constraints on their parameter spaces from vacuum stability and perturbativity as well as from LHC results and find that requiring vacuum stability up to the compositeness scale already imposes relevant constraints. A small part of parameter space around the classically conformal limit is stable up to the Planck scale. This is however already strongly disfavored by LHC results. In different limits, the models realize both (partially) composite-Higgs and (bosonic) technicolor models and a dynamical extension of the fundamental Goldstone-Higgs model. Therefore, they provide a general framework for exploring the phenomenology of composite dynamics.

KEywords: Beyond Standard Model, Global Symmetries, Spontaneous Symmetry Breaking, Technicolor and Composite Models

ARXIV EPRINT: 1711.10410 


\section{Contents}

1 Introduction 1

2 Minimal partially composite Higgs models $\quad 2$

2.1 Model I: elementary scalar doublet 3

2.2 Model II: SU(4) completion of the scalar sector 4

3 Model I: RG analysis and phenomenological constraints $\quad 6$

$\begin{array}{ll}3.1 \text { Vacuum, spectrum, and } \lambda_{H} & 6\end{array}$

$\begin{array}{llr}3.2 & \text { RG analysis and vacuum stability } & 9\end{array}$

$\begin{array}{lll}3.3 & \text { Additional source for the top-quark mass } & 10\end{array}$

$\begin{array}{lll}3.4 & \text { Phenomenology } & 12\end{array}$

$\begin{array}{lll}3.4 .1 & \text { Higgs couplings } & 12\end{array}$

$\begin{array}{lll}3.4 .2 & \text { Scalar production } & 14\end{array}$

$\begin{array}{lll}3.4 .3 & B^{0}-\overline{B^{0}} \text { mixing } & 15\end{array}$

4 Model II: RG analysis and phenomenological constraints 16

$\begin{array}{lll}4.1 \text { Vacuum and spectrum } & 16\end{array}$

$\begin{array}{lll}4.2 & \text { RG analysis } & 17\end{array}$

$\begin{array}{lll}4.3 & \text { Phenomenology } & 18\end{array}$

$\begin{array}{llr}5 & \text { Conclusions } & 20\end{array}$

$\begin{array}{ll}\text { A RG equations: elementary doublet } & 21\end{array}$

B RG equations: SU(4) multiplet of elementary scalars 22

\section{Introduction}

Partially composite-Higgs $(\mathrm{CH})$ models with underlying four-dimensional realizations in terms of new fermions coupled to elementary scalar multiplets via Yukawa interactions [1] were recently developed and studied in refs. [2-5]. These models feature dynamically induced electroweak symmetry breaking (EWSB) and a partially composite pseudo-Goldstone boson (pGB) Higgs. The elementary scalar multiplet provides masses for the SM fermions via SM-like Yukawa interactions [6] avoiding the complications of fermion partial compositeness [7] or extended-technicolor-type constructions [8-10]. The framework gives a unified description for various classes of models from (partially) $\mathrm{CH}$ [1] to (bosonic) technicolor (b)TC $[11,12]$.

However, such models may suffer from a low vacuum instability scale due to the enhancement of the top Yukawa coupling of the Higgs state as compared to the SM Higgs [13]. 
In this paper we, therefore, study the vacuum stability and perturbativity bounds on the parameter space of minimal partially $\mathrm{CH}(\mathrm{pCH})$ models $[2,3,5]$ and combine these selfconsistency conditions with current constraints from collider experiments. We show that even the requirement of stability up to the compositeness scale provides relevant constraints regardless of the particular UV realization. We also consider model extensions where a (small) part of the top-quark mass does not originate from the vacuum expectation value (vev) of the elementary scalar multiplet as a possibility to alleviate the vacuumstability bounds.

The two underlying concrete models we consider both consist of four Weyl fermions, $U_{L, R}, D_{L, R}$, transforming under a new $\mathrm{SU}(2)_{\mathrm{TC}}$ gauge group with the left-handed components forming a doublet, $\left(U_{\mathrm{L}}, D_{\mathrm{L}}\right)$, under the weak gauge group $\mathrm{SU}(2)_{\mathrm{w}}$. This is the minimal CH model with four-dimensional fermionic realisation [14], and the new fermion sector features an enhanced SU(4) global symmetry acting on the four Weyl fermions. The fermions are coupled to a multiplet of new elementary scalars via Yukawa interactions, and the dynamical condensation of the strongly interacting (techni)fermions is transmitted to the scalar multiplet via these Yukawa interactions. As a consequence, the fundamental scalar multiplet acquires a vev. We begin by considering a $\mathrm{SU}(2)_{\mathrm{w}}$ doublet of scalars $[2,3]$ and then study a full multiplet under the global SU(4) symmetry of the new fermion sector [5].

While the SM fermions obtain their masses via the vev of the elementary scalar multiplet, the weak boson masses originate from both the vev and the condensate in the composite sector. Consequently, the electroweak (EW) scale is given by

$$
v_{\mathrm{w}}^{2}=v^{2}+f^{2} \sin ^{2} \theta,
$$

where $v_{\mathrm{w}}=246 \mathrm{GeV}$, we denote the vev of the neutral CP-even component of the elementary Higgs by $v, f$ is the Goldstone-boson decay constant of the composite sector, and $\theta$ $(\pi / 2 \leq \theta \leq \pi)$ parameterises the vacuum misalignment.

\section{Minimal partially composite Higgs models}

We consider here the minimal four-dimensional strongly interacting fermionic sector allowing for a composite Goldstone Higgs boson. This is based on the gauge group $\mathrm{SU}(2)_{\mathrm{TC}}$ and contains four Weyl fermions transforming in the fundamental representation of the gauge group. The new fermion sector in isolation features a global SU(4) symmetry, which upon condensation breaks spontaneously to $\operatorname{Sp}(4)$ [15]. The left-handed fermions $\left(U_{L}, D_{L}\right)$ transform as a doublet under $\mathrm{SU}(2)_{\mathrm{w}}$, and the $\mathrm{EW}$ gauge group can be embedded into the $\mathrm{SU}(4)$ global symmetry via the left and right generators

$$
T_{\mathrm{L}}^{i}=\frac{1}{2}\left(\begin{array}{cc}
\sigma_{i} & 0 \\
0 & 0
\end{array}\right), T_{\mathrm{R}}^{i}=\frac{1}{2}\left(\begin{array}{cc}
0 & 0 \\
0 & -\sigma_{i}^{T}
\end{array}\right) .
$$

The $T_{\mathrm{L}}$ generators may be identified with those of the $\mathrm{SU}(2)_{\mathrm{w}}$ and $T_{\mathrm{R}}^{3}$ with the generator of hypercharge [16-19]. The five Goldstone bosons (GBs) associated to the breaking SU(4) $\rightarrow$ 


\begin{tabular}{|cccc|}
\hline & $\mathrm{SU}(2)_{\mathrm{TC}}$ & $\mathrm{SU}(2)_{\mathrm{W}}$ & $\mathrm{U}(1)_{Y}$ \\
\hline$Q_{\mathrm{L}}$ & $\square$ & $\square$ & 0 \\
$\widetilde{U}_{\mathrm{L}}$ & $\square$ & 1 & $-1 / 2$ \\
$\widetilde{D}_{\mathrm{L}}$ & $\square$ & 1 & $+1 / 2$ \\
\hline
\end{tabular}

Table 1. New fermion content.

$\mathrm{Sp}(4)$ then form an EW doublet and a singlet. We list the left-handed fermions and their quantum numbers using the notation $\widetilde{U}_{\mathrm{L}} \equiv \epsilon U_{R}^{*}$ in table 1 . The details of the construction of the effective description can be found e.g. in refs. [2, 5].

There are different possibilities to align the stability group, $\operatorname{Sp}(4)$, with respect to the EW gauge symmetry inside the SU(4). In particular, the EW group can be embedded completely within the stability group leading to unbroken EW symmetry. There are two inequivalent vacua, $E_{ \pm}$, corresponding to this orientation. On the other hand, it is possible that only the electromagnetic subgroup, $\mathrm{U}(1)_{Q}$, is unbroken as in TC models [20,21]. We denote the corresponding vacuum by $E_{\mathrm{B}}$. The different vacua can be represented as the matrices

$$
E_{ \pm}=\left(\begin{array}{cc}
\mathrm{i} \sigma_{2} & 0 \\
0 & \pm \mathrm{i} \sigma_{2}
\end{array}\right), \quad E_{B}=\left(\begin{array}{cc}
0 & 1 \\
-1 & 0
\end{array}\right) .
$$

In general, the vacuum is a superposition of an EW-preserving and the EW-breaking vacua, and can be written as

$$
E=\cos \theta E_{-}+\sin \theta E_{\mathrm{B}}
$$

The misalignment angle, $\theta$, is determined by the sources of explicit SU(4) breaking, and the relation between the composite scale and the EW scale is then given in terms of $\theta$ according to eq. (1.1).

We parameterise the composite Goldstone-boson degrees of freedom in the $\mathrm{SU}(4) / \mathrm{Sp}(4)$ coset by

$$
\Sigma=\exp \left(\frac{2 \sqrt{2} \mathrm{i}}{f} \Pi^{a} X^{a}\right) E,
$$

where the broken generators, $X^{a}$, with $a=1, \ldots, 5$, correspond to the vacuum $E$. The generators are explicitly given in ref. [17]. Furthermore, we introduce spurions, $P_{\alpha}, \widetilde{P}_{\alpha}$, such that $\operatorname{Tr}\left[P_{\alpha} \Sigma\right]$ and $\operatorname{Tr}\left[\widetilde{P}_{\alpha} \Sigma\right]$ transform as EW doublets with hypercharges $+1 / 2$ and $-1 / 2$, respectively. These can be explicitly written as

$$
\begin{aligned}
& 2 P_{1}=\delta_{i 1} \delta_{j 3}-\delta_{i 3} \delta_{j 1}, 2 P_{2}=\delta_{i 2} \delta_{j 3}-\delta_{i 3} \delta_{j 2} \\
& 2 \widetilde{P}_{1}=\delta_{i 1} \delta_{j 4}-\delta_{i 4} \delta_{j 1}, 2 \widetilde{P}_{2}=\delta_{i 2} \delta_{j 4}-\delta_{i 4} \delta_{j 2} .
\end{aligned}
$$

\subsection{Model I: elementary scalar doublet}

In addition to the composite pions, $\Sigma$, we introduce an (elementary) scalar multiplet. We begin by considering an $\mathrm{SU}(2)_{\mathrm{L}}$ doublet, $H_{\alpha}$, and we also introduce vector-like masses for 
the new fermions $[2,3]$ via the matrix $M$ :

$$
H_{\alpha}=\frac{1}{\sqrt{2}}\left(\begin{array}{c}
\sigma_{h}-\mathrm{i} \pi_{h}^{3} \\
-\left(\pi_{h}^{2}+\mathrm{i} \pi_{h}^{1}\right)
\end{array}\right), \quad M=\left(\begin{array}{cc}
m_{1} \epsilon & 0 \\
0 & -m_{2} \epsilon
\end{array}\right) .
$$

With this field content, the underlying Lagrangian describing the new strong sector and the elementary doublet can be written as

$$
\begin{aligned}
\mathcal{L}_{\mathrm{pCH}}= & \bar{Q} \mathrm{i} \not D Q+D_{\mu} H^{\dagger} D^{\mu} H-m_{H}^{2} H^{\dagger} H-\lambda_{H}\left(H^{\dagger} H\right)^{2} \\
& +\frac{1}{2} Q^{T} M Q-y_{U} H_{\alpha}\left(Q^{T} P_{\alpha} Q\right)-y_{D} \widetilde{H}_{\alpha}\left(Q^{T} \widetilde{P}_{\alpha} Q\right)+\text { h.c. }
\end{aligned}
$$

where $Q=\left(U_{\mathrm{L}}, D_{\mathrm{L}}, \widetilde{U}_{\mathrm{L}}, \widetilde{D}_{\mathrm{L}}\right), \widetilde{H}=\epsilon H^{*}$, and the antisymmetric contractions are kept implicit. The global SU(4) symmetry of the composite sector is in this case broken explicitly to $\mathrm{SU}(2)_{\mathrm{w}} \times \mathrm{U}(1)_{Y}$ by both the Yukawa and weak interactions, and the vector like masses in $M$. The Yukawa couplings with the SM fermions, restricting to the third generation of SM quarks, are

$$
\mathcal{L}_{\text {Yuk }}=-y_{t} \bar{q}_{\mathrm{L}} H t_{\mathrm{R}}-y_{b} \bar{q}_{\mathrm{L}} \widetilde{H} b_{\mathrm{R}}+\text { h.c., }
$$

where $q_{L}=\left(t_{L}, b_{L}\right)$. This model was considered in refs. [2,3] in the limit $\lambda_{H}=0$ but here we keep $\lambda_{H}$ throughout, since it is relevant for the vacuum stability, and since we do not assume necessarily any supersymmetric completion that would alter the running of $\lambda_{H}$ at high energies.

Below the condensation scale, $\Lambda_{\mathrm{TC}} \sim 4 \pi f$, eq. (2.7) yields the following lowest-order effective potential

$$
\begin{aligned}
V_{\text {eff }}^{0}= & 4 \pi f^{3} Z_{2}\left(\frac{1}{2} \operatorname{Tr}[M \Sigma]+y_{U} H_{\alpha} \operatorname{Tr}\left[P_{\alpha} \Sigma\right]+y_{D} \widetilde{H}_{\alpha} \operatorname{Tr}\left[\widetilde{P}_{\alpha} \Sigma\right]+\text { h.c. }\right) \\
& +m_{H}^{2} H^{\dagger} H+\lambda_{H}\left(H^{\dagger} H\right)^{2}
\end{aligned}
$$

where $Z_{2}$ is a non-perturbative $\mathcal{O}(1)$ constant, and we use the numerical value $Z_{2} \approx 1.5$ suggested by the lattice simulations ${ }^{1}[22]$. The EW gauge interactions contribute to the effective potential at the one-loop level, but the contribution is subleading as compared to the vector-like mass terms. It is, however, important for the model we describe in the next section.

\subsection{Model II: SU(4) completion of the scalar sector}

A model retaining the global SU(4) symmetry on the new fermion sector in the presence of the Yukawa interactions was proposed in ref. [5]. The elementary scalar doublet is extended to a complete two-index antisymmetric SU(4) representation, $\Phi$, allowing for the Yukawa interactions of the elementary scalars and the new fermions to remain SU(4) symmetric. Furthermore, the vector-like masses, $m_{1,2}$, of the new fermions above are then generated via the dynamically induced vev of the EW-singlet component of the scalar multiplet, $\Phi$.

\footnotetext{
${ }^{1}$ Ref. [22] gives $\left\langle Q^{T} Q\right\rangle^{1 / 3} / f=4.19(26)$ for the condensate, which then yields an estimate (with a factor 4 accounting for the trace): $Z_{2}=\frac{1}{4 \cdot 4 \pi}\left(\frac{\left\langle Q^{T} Q\right\rangle^{1 / 3}}{f}\right)^{3} \approx 1.5$.
} 
The misalignment of the vacuum, $\theta \neq 0$, required to give a mass to the would-be pGB Higgs boson may come solely from the SM sector and the scalar potential. In the following, we briefly outline the model and the construction of the effective low-energy Lagrangian, but we refer to ref. [5] for details.

The scalar multiplet, $\Phi$, can be conveniently parameterised in terms of the EW eigenstates. To this end, we write $H_{\alpha} \equiv \operatorname{Tr}\left[P_{\alpha} \Phi\right]$ and $\widetilde{H}_{\alpha} \equiv \operatorname{Tr}\left[\widetilde{P}_{\alpha} \Phi\right]$ that transform as EW doublets with hypercharges $+1 / 2$ and $-1 / 2$, respectively. The EW-singlet directions can be projected out with the following spurions:

$$
P_{1}^{\mathrm{S}}=\frac{1}{2}\left(\begin{array}{cc}
-\mathrm{i} \sigma_{2} & 0 \\
0 & 0
\end{array}\right), P_{2}^{\mathrm{S}}=\frac{1}{2}\left(\begin{array}{cc}
0 & 0 \\
0 & \mathrm{i} \sigma_{2}
\end{array}\right)
$$

Thus, we arrive at

$$
\Phi=\sum_{\alpha=1,2} P_{\alpha} H_{\alpha}+\widetilde{P}_{\alpha} \widetilde{H}_{\alpha}+P_{1}^{\mathrm{S}} S+P_{2}^{\mathrm{S}} S^{*}
$$

where $H$ is given in eq. (2.6), and $S=\frac{1}{\sqrt{2}}\left(S_{\mathrm{R}}+\mathrm{i} S_{\mathrm{I}}\right)$ parameterises the EW-singlet scalars. The relevant Lagrangian, restricting to third generation of SM quarks, is then

$$
\begin{aligned}
\mathcal{L}_{\mathrm{pCH}_{2}}= & \bar{Q} \mathrm{i} \not D Q+\operatorname{Tr}\left[D_{\mu} \Phi^{\dagger} D^{\mu} \Phi\right]-m_{\Phi}^{2} \operatorname{Tr}\left[\Phi^{\dagger} \Phi\right]-\lambda_{\Phi} \operatorname{Tr}\left[\Phi^{\dagger} \Phi\right]^{2} \\
& -y_{Q} Q^{T} \Phi Q-y_{t} \bar{q}_{\mathrm{L}} H t_{\mathrm{R}}-y_{b} \bar{q}_{\mathrm{L}} \widetilde{H} b_{\mathrm{R}}+\text { h.c. }
\end{aligned}
$$

Below the condensation scale, we have the lowest-order effective potential

$$
V_{\text {eff }}^{0}=4 \pi f^{3} Z_{2}\left(y_{Q} \operatorname{Tr}[\Phi \Sigma]+\text { h.c. }\right)+m_{\Phi}^{2} \operatorname{Tr}\left[\Phi^{\dagger} \Phi\right]+\lambda_{\Phi} \operatorname{Tr}\left[\Phi^{\dagger} \Phi\right]^{2} .
$$

At this level, the global symmetry is broken only spontaneously by the condensation in the strong sector. Therefore, in order to misalign the vacuum with respect to EW symmetry and to give a mass to one of the GB's to be identified with the Higgs, we need to consider sources that break the global symmetry explicitly. To this end, we include the leading EW contribution in the effective potential $[23,24]$

$$
V_{\text {gauge }}=-C_{g}\left[g^{2} f^{4} \sum_{i=1}^{3} \operatorname{Tr}\left(T_{\mathrm{L}}^{i} \Sigma\left(T_{\mathrm{L}}^{i} \Sigma\right)^{*}\right)+g^{\prime 2} f^{4} \operatorname{Tr}\left(T_{\mathrm{R}}^{3} \Sigma\left(T_{\mathrm{R}}^{3} \Sigma\right)^{*}\right)\right],
$$

where $C_{g}$ is a positive $\mathcal{O}(1)$ loop factor. Differently from ref. [18], the top quark interactions are subleading in the vacuum determination, since the top quark only couples to the weakly interacting fundamental sector. We add another source of explicit breaking of the global symmetry by splitting of the masses of the EW-doublet and singlet components of the scalar multiplet, $\Phi$ :

$$
V_{\delta m^{2}}=2 \delta m^{2} \operatorname{Tr}\left[P_{i}^{S} \Phi\right] \operatorname{Tr}\left[P_{i}^{S} \Phi\right]^{*}=\frac{1}{2} \delta m^{2}\left(S_{\mathrm{R}}^{2}+S_{\mathrm{I}}^{2}\right) .
$$

As shown in ref. [5], these two sources are enough to achieve a non-trivial vacuum alignment, and therefore we need not introduce further explicit breaking sources directly on the new fermion sector. 


\section{Model I: RG analysis and phenomenological constraints}

\subsection{Vacuum, spectrum, and $\lambda_{H}$}

The effective potential of eq. (2.9) as a function of $\sigma_{h}$ and $\theta$ reads

$$
V_{\text {eff }}=8 \pi f^{3} Z_{2}\left(m_{12} c_{\theta}-\frac{y_{U D}}{\sqrt{2}} \sigma_{h} s_{\theta}\right)+\frac{1}{2} m_{H}^{2} \sigma_{h}^{2}+\frac{\lambda_{H}}{4} \sigma_{h}^{4},
$$

where $m_{12} \equiv m_{1}+m_{2}$ and $y_{U D} \equiv y_{U}+y_{D}$. We use the short-hand notations $s_{x} \equiv \sin x$, $c_{x} \equiv \cos x$, and $t_{x} \equiv \tan x$. Further, we define the parameter $m_{\lambda}^{2} \equiv m_{H}^{2}+\lambda_{H} v^{2}$, the form factor $F_{0}=4 \pi Z_{2}$ and trade $v$ for the angle $\beta$ via

$$
t_{\beta} \equiv \frac{v}{f s_{\theta}} .
$$

The vacuum conditions then read

$$
\begin{aligned}
& 0=\left.\frac{\partial V_{\mathrm{eff}}}{\partial \sigma_{h}}\right|_{\sigma_{h}=v}=-\sqrt{2} F_{0} y_{U D} f^{3} s_{\theta}+m_{\lambda}^{2} v, \\
& 0=\left.\frac{\partial V_{\mathrm{eff}}}{\partial \theta}\right|_{\sigma_{h}=v}=-2 F_{0} f^{3}\left(m_{12} s_{\theta}+\frac{y_{U D}}{\sqrt{2}} v c_{\theta}\right) .
\end{aligned}
$$

These yield the parameter constraint equations

$$
y_{U D}=\frac{t_{\beta} m_{\lambda}^{2}}{\sqrt{2} f^{2} F_{0}}, \quad m_{12}=-\frac{c_{\theta} m_{\lambda}^{2} t_{\beta}^{2}}{2 F_{0} f} .
$$

For $t_{\beta} \gtrsim 1$, the first equation limits the mass parameter to $m_{H}^{2} \lesssim 4 \pi f^{2}$ as long as $y_{U D} \lesssim 1$. If the latter limit on $y_{U D}$ is not satisfied, then these large Yukawa couplings will drive the scalar quartic negative at very low scales. Indeed we find that $y_{U D} \ll 1$ for all the viable parameter space that we consider, and this condition is thus automatically satisfied.

The model features seven parameters relevant to our study, ${ }^{2} y_{U D}, m_{12}, \lambda_{H}, s_{\theta}, t_{\beta}, f$, $m_{H}^{2}$, and four constraint equations including the two vacuum conditions in eq. (3.3), the EW scale eq. (1.1), and the Higgs mass, whose expression is shown below in eq. (3.9). We take $s_{\theta}, t_{\beta}$ and $m_{H}^{2}$ as free parameters.

The Yukawa coupling between the top quark and the elementary doublet is enhanced with respect to the SM value by

$$
y_{t}=y_{t}^{\mathrm{SM}} / s_{\beta} .
$$

For a given value of the quartic coupling, $\lambda_{H}$, in eq. (2.7), this implies a lower limit on $t_{\beta}$ in order to avoid vacuum instability above the EWSB scale and below the Planck scale. We discuss this issue in detail in section 3.2.

The charged pion and neutral CP-even scalar mass matrices, in the bases $\left(\pi_{h}^{+}, \Pi^{+}\right)$, $\left(\sigma_{h}, \Pi^{4}\right)$, resp., can be written as

$$
M_{\pi^{+}}^{2}=m_{\lambda}^{2}\left(\begin{array}{cc}
1 & t_{\beta} \\
t_{\beta} & t_{\beta}^{2}
\end{array}\right), \quad M_{h}^{2}=m_{\lambda}^{2}\left(\begin{array}{cc}
1+\delta & -c_{\theta} t_{\beta} \\
-c_{\theta} t_{\beta} & t_{\beta}^{2}
\end{array}\right),
$$

\footnotetext{
${ }^{2}$ Only the combinations $m_{12}$ and $y_{U D}$ enter into the vacuum equations and the effect of $y_{U}, y_{D}$ on the running of the couplings is negligible in the parameter space considered.
} 
where $\delta \equiv 2 \frac{\lambda_{H} v^{2}}{m_{\lambda}^{2}}$. The mass of the heavy neutral pion, $\pi^{0}$, coincides with the mass of the charged ones.

The CP-even mass eigenstates are given in terms of the interaction eigenstates by

$$
h_{1}=c_{\alpha} \sigma_{h}-s_{\alpha} \Pi^{4}, \quad h_{2}=s_{\alpha} \sigma_{h}+c_{\alpha} \Pi^{4},
$$

with

$$
t_{2 \alpha}=\frac{2 t_{\beta} c_{\theta}}{1-t_{\beta}^{2}+\delta}
$$

The corresponding masses of the neutral and charged eigenstates are

$$
\begin{aligned}
m_{h_{1,2}}^{2} & =\frac{m_{\lambda}^{2}}{2}\left[1+t_{\beta}^{2}+\delta \pm\left(2 c_{\theta} t_{\beta} s_{2 \alpha}+\left(1-t_{\beta}^{2}+\delta\right) c_{2 \alpha}\right)\right], \\
m_{\pi^{ \pm}, 0}^{2} & =m_{\lambda}^{2} / c_{\beta}^{2}, \quad m_{\Pi_{5}}^{2}=t_{\beta}^{2} m_{\lambda}^{2} .
\end{aligned}
$$

Using eq. (3.8) and expanding in $s_{\theta}^{2}$ and $t_{\beta}^{-2}$, we then find

$$
m_{h_{1}}^{2} \simeq m_{\lambda}^{2}\left(s_{\theta}^{2}+\left(1-t_{\beta}^{-2}\right) \delta\right), \quad m_{h_{2}}^{2} \simeq m_{\lambda}^{2}\left(1+t_{\beta}^{2}\right), \quad m_{\pi^{ \pm}, 0}^{2}=m_{\lambda}^{2}\left(1+t_{\beta}^{2}\right) .
$$

We now identify the lightest of the neutral CP-even eigenstates with the $125-\mathrm{GeV}$ Higgs boson. In most of parameter space this is the $h_{1}$ state, but for $1+\delta>t_{\beta}^{2}$ it is instead $h_{2}$ as seen from eq. (3.6). We denote the heavier CP-even eigenstate by $\mathcal{H}$.

We show the mass of $\mathcal{H}$ for fixed $m_{H}^{2} / f^{2}=0,1$ in the $\left(s_{\theta}, t_{\beta}\right)$ plane in the left panel of figure 1 . The simple scaling of the heavy masses with $t_{\beta}$, roughly independent of $s_{\theta}$, is apparent in the $m_{H}^{2} / f^{2}=0$ case (blue lines). Instead the dependence on $s_{\theta}$ in the case $m_{H}^{2} / f^{2}=1$ (red dotted lines) follows from the vev condition eq. (1.1) implying the scaling $m_{\lambda}^{2} \simeq m_{H}^{2} \simeq v_{\mathrm{EW}}^{2} s_{\theta}^{-2} t_{\beta}^{-2}$ which cancels the $t_{\beta}^{2}$ factor in the heavy mass formulas. The mass of the heavy pion triplet nearly coincides with the mass of the heavier CP-even state for the parameters we consider, and we have omitted that for clarity in the plot.

Using the experimental value of the Higgs mass and fixing the mass parameter to $m_{H}^{2}=0\left(m_{H}^{2}=f^{2}\right)$, we show on the left panel of figure 2 with blue (yellow) contours the value of $\lambda_{H}$ in $\left(s_{\theta}, t_{\beta}\right)$ plane. The figure demonstrates that the value of $\lambda_{H}$ may only be increased slightly beyond its value in the SM $\lambda_{\mathrm{SM}} \approx 0.129$ unless $t_{\beta}$ is very small. Since for large $t_{\beta}$, the light Higgs mass is a sum of two manifestly positive contributions, cf. eq. (3.10), the scalar quartic coupling is bounded for a given value of $t_{\beta}$ to $\lambda_{H} \lesssim \lambda_{\mathrm{SM}} / s_{\beta}^{2}$, and the bound is only reached in the limit $m_{H}^{2} \rightarrow 0, s_{\theta} \rightarrow 0$. Furthermore, for $t_{\beta} \rightarrow \infty$, this bound reduces to the SM value. On the right panel of figure 2, we show the contour lines in the $\left(t_{\beta}, \lambda_{H}\right)$ plane for different values of $\Lambda_{\mathrm{TC}}=4 \pi f$ in $\mathrm{TeV}$ having fixed the Higgs mass, and the mass of the heavier eigenstate to $1 \mathrm{TeV}$ for concreteness. In the lower shaded region, $\lambda_{H}<0$ and this is excluded due to vacuum instability. The boundary of the upper blue shaded region shows the maximum allowed value of $\lambda_{H}$ for which the correct Higgs mass can still be achieved as a function of $t_{\beta}$. The $\Lambda_{\mathrm{TC}} \rightarrow \infty$ contours lie very close to the $\Lambda_{\mathrm{TC}}=50 \mathrm{TeV}$ contour. This boundary is almost independent of the fixed value of the heavy eigenstate mass, and the blue shaded region is thus inaccessible.

The accessible values of $\lambda_{H}$ shown in figure 2, together with the enhanced top Yukawa in eq. (3.5), make it clear that it is important to consider the vacuum stability constraints on the model. 

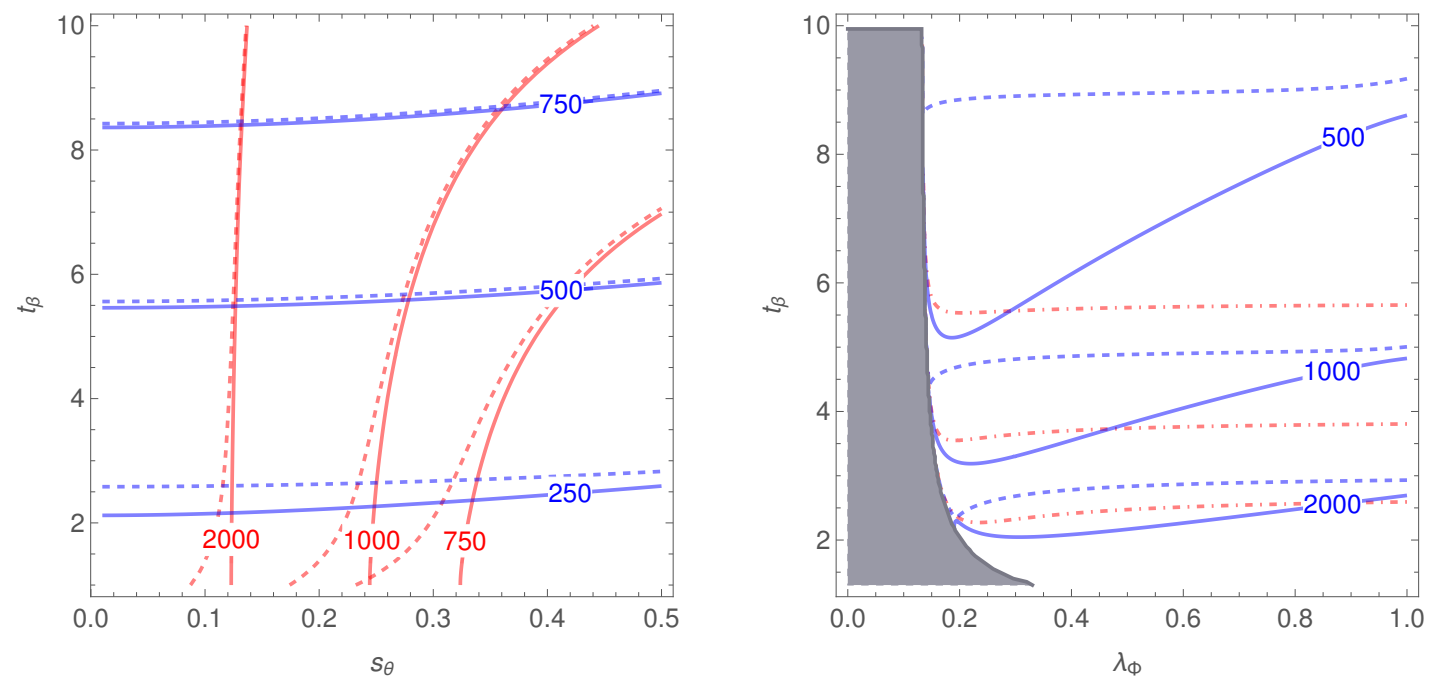

Figure 1. Left panel: model I spectrum. The blue (red) solid contours show the mass of the heavier CP-even state, $\mathcal{H}$, in $\mathrm{GeV}$ for fixed $m_{H}^{2} / f^{2}=0\left(m_{H}^{2} / f^{2}=1\right)$. The dashed curves show the mass of the pseudoscalar $\Pi_{5}$ for the same scenarios. Right panel: model II spectrum. The solid (dashed) blue lines show the mass contours $(500 \mathrm{GeV}, 1000 \mathrm{GeV}, 2000 \mathrm{GeV})$ of $m_{h_{2}}\left(m_{h_{3}}\right)$. The mass of the heavy pion triplet follows closely the contours of $m_{h_{3}}$, and we have omitted those for clarity. The dot-dashed red lines show the same mass contours for the non-pGB mass eigenstate of the two mixing pseudoscalar states, $\Pi_{5}$ and $S_{\mathrm{I}}$. We have fixed $s_{\theta}=0.1$.
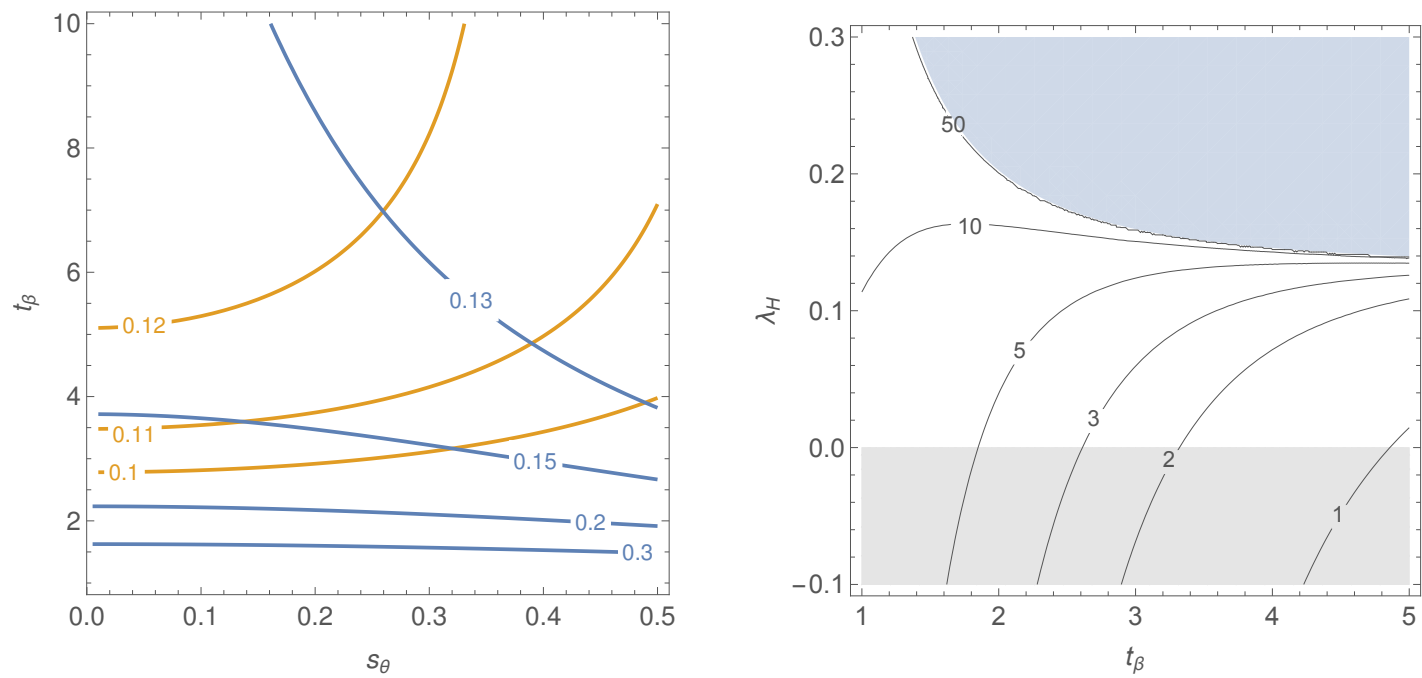

Figure 2. Left panel: values of $\lambda_{H}$. Blue (yellow) contours represent the case $m_{H}^{2} / f^{2}=0$ $\left(m_{H}^{2} / f^{2}=1\right)$. Right panel: the contours show the value of $\Lambda_{\mathrm{TC}}=4 \pi f$ in $\mathrm{TeV}$ corresponding to a $1 \mathrm{TeV}$ heavy Higgs mass. The region $\lambda_{H}<0$ is excluded by the requirement of stability of the potential. For $\Lambda_{\mathrm{TC}} \rightarrow \infty$ the curves lie on top of the $\Lambda_{\mathrm{TC}}=50 \mathrm{TeV}$ contour so the shaded area results in no viable solutions. More details in the text. 

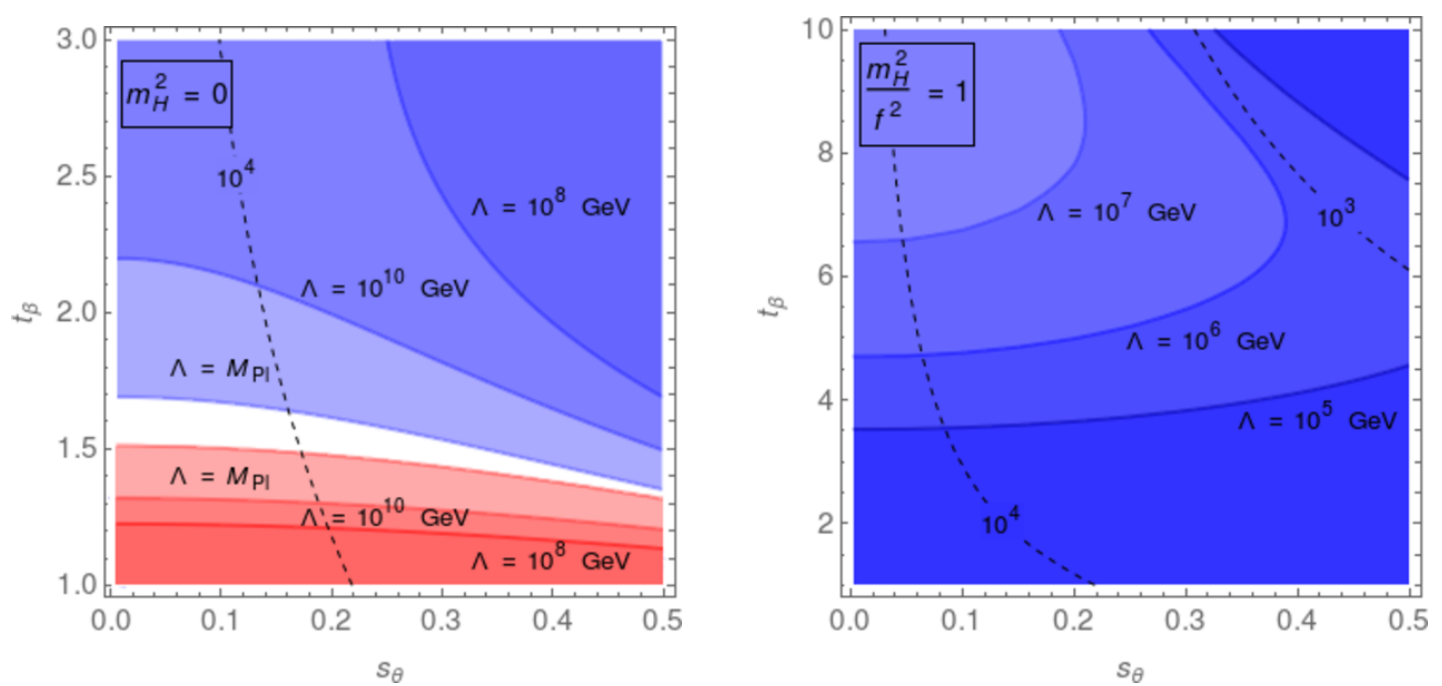

Figure 3. The blue (red) regions illustrate the vacuum instability (non-perturbativity) scale. The dashed lines show the values of $\Lambda_{\mathrm{TC}}=4 \pi f$ in GeV. Left panel: $m_{H}^{2}=0$. On the boundaries of the regions, the vacuum is stable (couplings pertubative) up to $\Lambda=M_{\mathrm{Pl}}=$ $1.22 \cdot 10^{19} \mathrm{GeV}, 10^{10} \mathrm{GeV}, 10^{8} \mathrm{GeV}$. Right panel: $m_{H}^{2} / f^{2}=1$. On the boundaries of the regions, the vacuum is stable up to $\Lambda=10^{7} \mathrm{GeV}, 10^{6} \mathrm{GeV}, 10^{5} \mathrm{GeV}$.

\subsection{RG analysis and vacuum stability}

The running of $\lambda_{H}$ is driven dominantly by the top loop, as in the SM. However, compared to the SM, the top Yukawa coupling of the elementary interaction eigenstate, $\sigma_{h}$, is enhanced by $y_{t}=y_{t}^{\mathrm{SM}} / s_{\beta}$. Therefore $\lambda_{H}$ will run negative faster than in the SM for the same weak scale initial value, and the vacuum of the model will, thus, not be stable in all of parameter space. The problem is similar to that in bTC [13] and in type-I two-Higgs-doublet models (2HDM).

In appendix A, we tabulate the one-loop beta functions for the $\mathrm{bTC} / \mathrm{pCH}$ framework with $N_{\mathrm{F}}$ new $\mathrm{SU}(2)_{\mathrm{L}}$-doublet fermionic fields, $Q_{\mathrm{L}}=\left(U_{\mathrm{L}}, D_{\mathrm{L}}\right)$, transforming under the representation $R_{\mathrm{F}}$ of the new strong gauge group. These fields will couple to the elementary Higgs doublet, $H$, through Yukawa-like interactions with couplings $y_{U}$ and $y_{D}$.

In the numerical analysis we assume that the top quark Yukawa and the EW gauge couplings run as in the SM below the condensation scale $\Lambda_{\mathrm{TC}}$, and we neglect the threshold effects on the quartic coupling running. We fix the initial values of the SM gauge couplings at $\mu_{Z}=m_{Z}$, and the value of the SM top Yukawa from $y_{t}^{\mathrm{SM}}\left(m_{t}\right)$ as in [25]. Furthermore, at the condensation scale, $\Lambda_{\mathrm{TC}}$, we fix the TC gauge coupling to the estimate of the critical value [26], $\alpha_{\mathrm{TC}}^{\mathrm{c}}=\frac{\kappa}{C_{2}\left(R_{F}\right)}$, where $\kappa \sim \mathcal{O}(1)$, and we assume identical Yukawa couplings of the $U, D$ technifermions $y_{U}\left(\Lambda_{\mathrm{TC}}\right)=y_{D}\left(\Lambda_{\mathrm{TC}}\right) \equiv y_{Q}^{0}$. However, we note that since $y_{U D} \ll 1$ throughout parameter space consistent with the vacuum and Higgs mass conditions, the contribution of the new fermions to the running of the quartic coupling is negligible.

In figure 3 , we show the vacuum instability and perturbativity scales, $\Lambda_{\text {stab }}, \Lambda_{\text {pert }}$, resp., defined through $\lambda_{H}\left(\Lambda_{\text {stab }}\right)=0, \lambda_{H}\left(\Lambda_{\text {pert }}\right)=4 \pi$, in the $s_{\theta}, t_{\beta}$ plane for two different values of $m_{H}^{2} / f^{2}=0$ and $m_{H}^{2} / f^{2}=1$. Small values $m_{H}^{2} / f^{2} \ll 1$ correspond to large values of 


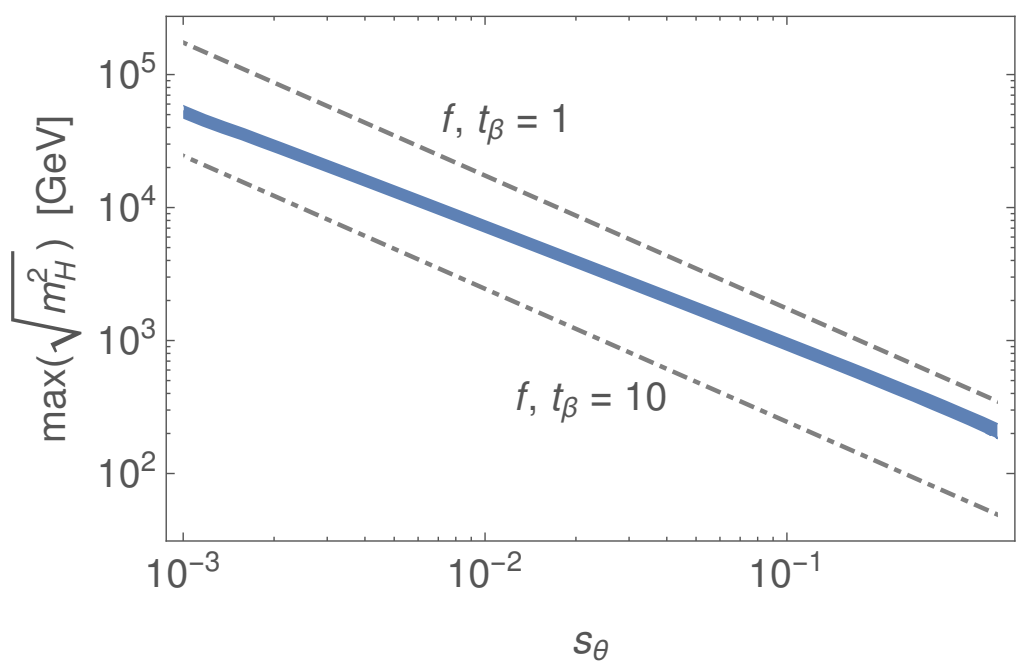

Figure 4. The blue band shows the maximum allowed value of $\sqrt{m_{H}^{2}}$ as a function of $s_{\theta}$ if stability at least up to the compositeness scale, $\Lambda_{\mathrm{TC}}=4 \pi f$, is required and $t_{\beta}$ is varied within the range $1 \ldots 10$. The dashed (dot-dashed) line shows $f$ as a function of $s_{\theta}$ for constant value of $t_{\beta}=1$ $\left(t_{\beta}=10\right)$.

$\lambda_{H}$ as is evident from the vacuum equations and yield a larger vacuum instability scale. As seen in the left panel of figure 3 , there is a narrow window in parameter space with vacuum stability and perturbativity up to the Planck scale for relatively small $t_{\beta}$. For even smaller values of $t_{\beta}$, the quartic becomes non-perturbative, whereas for larger values of $t_{\beta}$ the initial value of $\lambda_{H}$ decreases, while the top coupling is still enhanced leading to vacuum instability at lower scales. Increasing $m_{H}^{2} / f^{2}$ lowers the viable values of $\lambda_{H}$, and this window is lost as seen in the right panel of figure 3 .

The same increase in scale happens when $s_{\theta}$ is reduced with $m_{H}^{2}$ and $t_{\beta}$ fixed. This corresponds to decreasing of $v / f$ and thus from the vacuum conditions and the Higgs mass condition increasing $\lambda_{H}$.

Overall, demanding a stable vacuum to high scales requires tuning $m_{H}^{2} / f^{2}$ small. This implies at least a modest hierarchy between the scalar mass parameter and the compositeness scale. On the other hand, increasing the (absolute value of the) scalar mass parameter, $m_{H}^{2}$, with respect to the SM value requires tuning $s_{\theta} \ll 1$ small. This must arise from balancing two sources of unrelated physics. In figure 4, we show the maximum allowed values of $m_{H}^{2}$ as a function of $s_{\theta}$ demanding vacuum stability at least up to the compositeness scale, $\Lambda_{\mathrm{TC}}=4 \pi f$. This constrains the maximum possible $m_{H}^{2}$ regardless of the UV completion above the compositeness scale. We emphasize that while tuning $s_{\theta} \ll 1$ allows for larger values of $m_{H}^{2}$, it does not increase the maximum possible value of $m_{H}^{2} / f^{2}$. For example for $s_{\theta}=0.1$, and $t_{\beta}=1\left(t_{\beta}=10\right)$ we find $\max \left(\frac{m_{H}^{2}}{f^{2}}\right) \approx 0.3$ (14.5), whereas for $s_{\theta}=10^{-3}$, the corresponding value is $0.1(5.7)$.

\subsection{Additional source for the top-quark mass}

As seen in the previous RG analysis, in the simplest partially composite scenarios, the topYukawa coupling is enhanced with respect to the SM driving the running quartic coupling 
to negative values before the Planck scale in most of parameter space. As seen in figure 3, there is a small region where the weak scale value of the quartic itself is sufficiently enhanced with respect to the SM Higgs value, to counter balance the enhanced top Yukawa. A way to reduce the constraints from vacuum stability is to assume the top quark mass is not entirely due to the elementary scalar vev, but acquires a contribution from some still unspecified dynamics. This implies a reduction of the top Yukawa coupling of $\sigma_{h}$ in eq. (3.5).

To this end, we start by adding a four-fermion operator between the top and the techniquarks

$$
\mathcal{L}_{4 \mathrm{f}} \sim-\frac{Y_{t} Y_{U}}{\Lambda_{t}^{2}}\left(\bar{q}_{\mathrm{L}} t_{\mathrm{R}}\right)_{\alpha}^{\dagger}\left(Q^{T} P_{\alpha} Q\right)+\text { h.c. }
$$

We discuss the possible sources of the above four-fermion operator in the end of the section.

Upon the condensation of the techniquarks, this yields a contribution to the top mass, i.e.

$$
\mathcal{L}_{4 \mathrm{f}} \sim-4 \pi f^{3} Z_{2} \frac{Y_{t} Y_{U}}{\Lambda_{t}^{2}} \operatorname{Tr}\left[P_{1} \Sigma\right] \bar{t} t=-y_{t}^{\prime} f s_{\theta} \bar{t} t+\ldots
$$

where

$$
y_{t}^{\prime} \equiv \frac{4 \pi f^{2} Y_{t} Y_{U} Z_{2}}{\Lambda_{t}^{2}}
$$

Furthermore, this gives a contribution to the effective potential via the top loop

$$
V_{\text {top }}=-C_{t} y_{t}^{\prime 2} f^{4}\left|\operatorname{Tr}\left[P_{\alpha} \Sigma\right]\right|^{2}=-C_{t} y_{t}^{\prime 2} f^{4} s_{\theta}^{2}+\ldots
$$

Ignoring the subdominant EW-gauge contributions, the effective potential becomes

$$
V_{\text {eff,top }}=V_{\text {eff }}+V_{\text {top }}
$$

where $V_{\text {eff }}$ is given in eq. (2.9). Defining $\xi_{t}$ as the fraction of the top mass originated from this new contribution,

$$
y_{t}^{\prime} f s_{\theta} \equiv \xi_{t} m_{t},
$$

the vacuum condition in $\theta$ is changed to

$$
0=\left.\frac{\partial V_{\text {eff,top }}}{\partial \theta}\right|_{\mathrm{vac}}=\left.\frac{\partial V_{\text {eff }}}{\partial \theta}\right|_{\mathrm{vac}}-\frac{2 f^{2} C_{t} \xi_{t}^{2} m_{t}^{2}}{t_{\theta}}
$$

The neutral mass matrix becomes

$$
M_{h, \text { top }}^{2}=m_{\lambda}^{2}\left(\begin{array}{cc}
1+\delta & -c_{\theta} t_{\beta} \\
-c_{\theta} t_{\beta} & t_{\beta}^{2}+2 C_{t} \xi_{t}^{2} m_{t}^{2} / m_{\lambda}^{2}
\end{array}\right) .
$$

The effect of the additional contribution to the top mass has only a tiny effect on the vacuum alignment and the scalar mass eigenvalues in most of the parameter space. Therefore, for subdominant contribution to the top mass, the main effect is just a small reduction of the top-Yukawa coupling, with eq. (3.5) modified into

$$
y_{t}=y_{t}^{\mathrm{SM}}\left(1-\xi_{t}\right) / s_{\beta}
$$



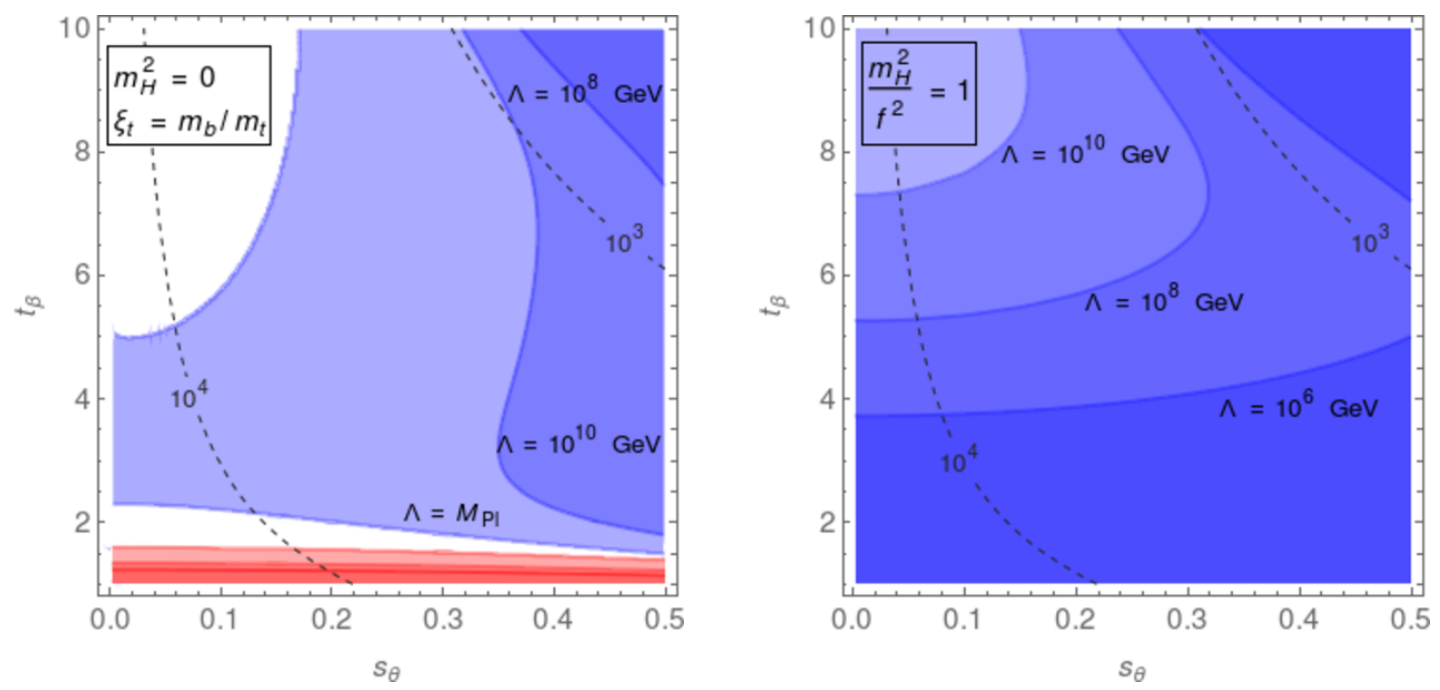

Figure 5. Corresponding plots to figure 3 with $\xi_{t}=m_{b} / m_{t}$. On the unshaded area $\Lambda \geq M_{\mathrm{Pl}}$. The dashed lines show the values of $4 \pi f$ in $\mathrm{GeV}$.

However, in the simplest UV-completion scenarios of the four-fermion operator, the effective treatment is valid only if the contribution to the top mass is very small.

A simple possibility to generate the four-fermion operator in eq. (3.11) is to add an additional heavy scalar doublet that couples to the top quark and to the new fermions and can be integrated out above the compositeness scale $\Lambda_{\mathrm{TC}} \sim 4 \pi f$ [11]. Another possibility would be devising extended-technicolor (ETC) interactions $[8,9]$. As opposed to ordinary TC models, ETC would only have to give a small contribution to the top mass. This would allow a higher ETC scale and thus alleviate the constraints from flavour-changing neutral currents. It would also potentially simplify the ETC construction, having only to involve the third generation as in e.g. ref. [27]. A small reduction (at the per cent level), such as provided by a new isospin-symmetric sector giving equal mass contributions to both top and bottom and providing all of the bottom mass already soften the rapid decrease of the quartic coupling via the $\mathrm{RG}$, thus enlarging the allowed range of parameter space constrained by vacuum instability. The stability plots for the value $\xi_{t}=m_{b} / m_{t} \simeq 0.025$ are shown in figure 5 . The figures thus correspond to those in figure 3 , except that part of the top mass now originates from the four-fermion operator above. The situation is in fact similar to the SM case where the uncertainty in the top mass could, in fact, bring the SM from meta-stable to stable.

\subsection{Phenomenology}

In this section we study constraints on the model arising from the modified Higgs couplings, from searches for new heavy scalars, and the new contributions to $B$-meson mixing.

\subsubsection{Higgs couplings}

To study the couplings of the $125-\mathrm{GeV}$ Higgs state, we first define the $\kappa_{f, i}$ and $\kappa_{V, i}$ coefficients, parameterising the couplings of the mass eigenstate $h_{i}, i=1,2$ to SM fermions, $f$, 
and vector bosons, $V$, relative to the SM Higgs boson couplings

$$
\kappa_{f, i} \equiv \frac{g_{h_{i} \bar{f} f}}{g_{h \bar{f} f}^{\mathrm{SM}}}, \quad \kappa_{V, i} \equiv \frac{g_{h_{i} V V}}{g_{h V V}^{\mathrm{SM}}}, \quad i=1,2 .
$$

The $h_{1}$ coefficients and their $s_{\theta}^{2}, t_{\beta}^{-2} \ll 1$ expansions to second order are given by

$$
\begin{gathered}
\kappa_{V, 1}=c_{\alpha} s_{\beta}-s_{\alpha} c_{\beta} c_{\theta} \rightarrow 1-\frac{1}{2} s_{\theta}^{2} t_{\beta}^{-2} \\
\kappa_{f, 1}=c_{\alpha} / s_{\beta} \rightarrow 1+\frac{1}{2} s_{\theta}^{2} t_{\beta}^{-2}-t_{\beta}^{-4} \delta .
\end{gathered}
$$

The $h_{2}$ coefficients and their expansions to first order are given by

$$
\begin{aligned}
& \kappa_{V, 2}=s_{\alpha} s_{\beta}+c_{\alpha} c_{\beta} c_{\theta} \rightarrow \delta t_{\beta}^{-3}, \\
& \kappa_{f, 2}=s_{\alpha} / s_{\beta} \rightarrow t_{\beta}^{-1}+\delta t_{\beta}^{-3}-\frac{1}{2} t_{\beta}^{-1} s_{\theta}^{2} .
\end{aligned}
$$

The exact expressions above coincide with those derived in ref. [2] and with those in a type I $2 \mathrm{HDM}$ except for the appearance of $c_{\theta}$.

Since the $125-\mathrm{GeV}$ Higgs state is identified with $h_{2}$ instead of $h_{1}$ in the small part of parameter space with $1+\delta>t_{\beta}^{2}$ as discussed below eq. (3.10), we use $\kappa_{f}$ and $\kappa_{V}$ to denote the coupling coefficients of the $125-\mathrm{GeV}$ Higgs state in all of parameter space. We show the values of these coefficients in the left panel of figure 6 for $m_{H}^{2}=0(\delta=2)$ together with the region disfavoured at the $2 \sigma$ level by LHC measurements of the Higgs couplings from [28]. In the parameter space shown, the deviation of $\kappa_{f}$ from unity is nearly independent of $s_{\theta}$ as follows from eqs. (3.21) and (3.22) when $\delta$ is sufficiently large. In the very small $t_{\beta}$ region, the expansions are no longer applicable.

The figure demonstrates how most of the considered parameter space is still viable, but interestingly the classically scale-invariant region in figure 3 with vacuum stability up to the Planck scale is just above what is currently probed by LHC Higgs coupling measurements. For larger values of $m_{H}^{2}$ (smaller $\delta$ ) the deviations from the SM value are even smaller.

In the case where we allow for a small part, $\xi_{t}$, of the top mass coming from a new sector encoded in the four-fermion interaction in eq. (3.11), the coupling of the top quark to Higgs is only slightly further modified:

$$
\kappa_{t}=-\xi_{t} \frac{s_{\alpha} c_{\theta}}{c_{\beta}}+\left(1-\xi_{t}\right) \frac{c_{\alpha}}{s_{\beta}} .
$$

A motivated possibility would be a new isospin-symmetric sector providing equal mass contributions to the third generation and providing all of the bottom mass. In this case $\xi_{t}=m_{b} / m_{t}$, and this would result in a significant modification in the couplings of the partially composite Higgs to bottom quarks:

$$
\kappa_{b}=-\frac{s_{\alpha} c_{\theta}}{c_{\beta}} .
$$

Interestingly, the measured value of this coupling by ATLAS and CMS combined is $\left|\kappa_{b}\right|=$ $0.57 \pm 0.16[28]$ and indicates a reduction of approximately $2.7 \sigma$ with respect to the SM 

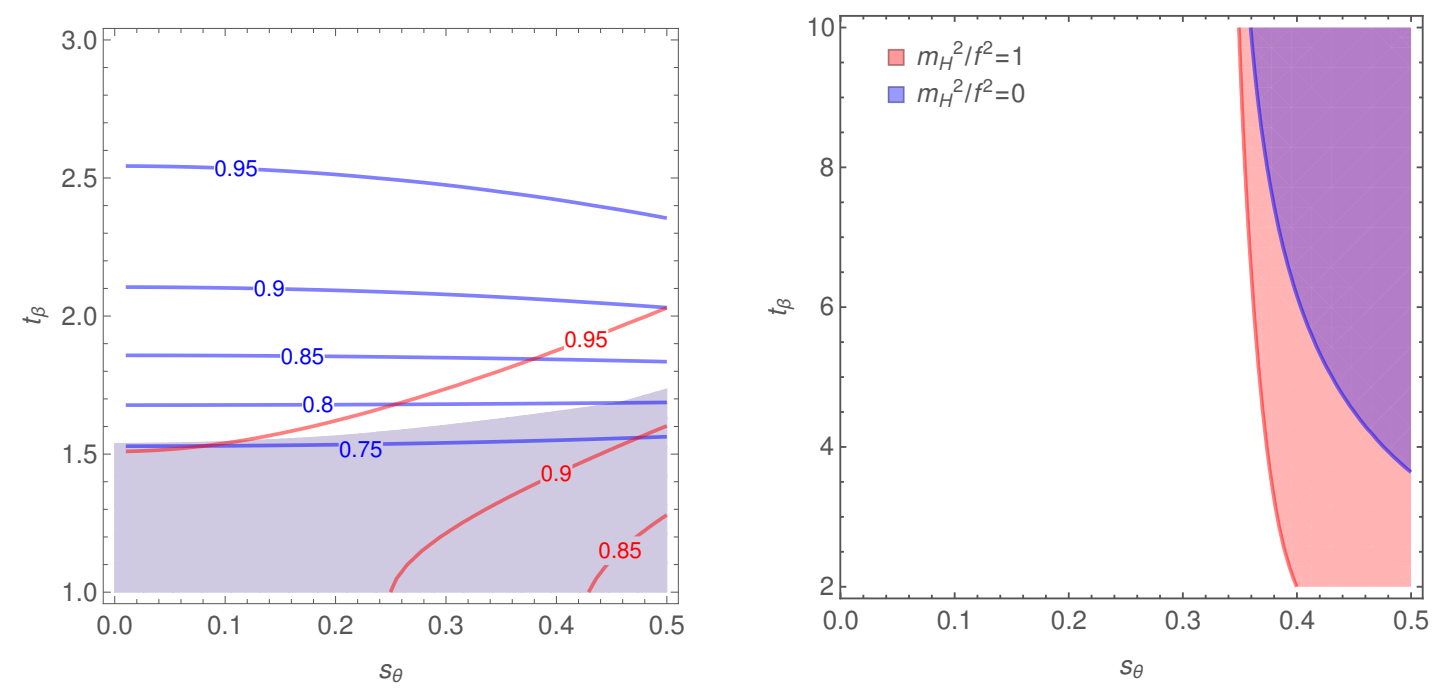

Figure 6. Left panel: the blue (red) curves show $\left|\kappa_{t}\right|\left(\left|\kappa_{V}\right|\right)$ for $m_{H}^{2}=0$ case. The shaded region shows the $2 \sigma$ exclusion from the two-parameter $\kappa_{f}, \kappa_{V}$ fit [28]. Right panel: region in which $\kappa_{b}$ is within $2 \sigma$ of the measured value $\left|\kappa_{b}\right|=0.57 \pm 0.16$, improved w.r.t. the SM prediction.

prediction according to the estimate which allows beyond-the-SM contribution in the loops. In the right panel of figure 6 , we show the favoured region in which $\left|\kappa_{b}\right|$ is within the $2 \sigma$ region of the measured value, for $m_{H}^{2}=f^{2}$, and $m_{H}^{2}=0$. This reduction favours large values of $s_{\theta}$, which implies a tension with the requirement of vacuum stability up to high scales.

\subsubsection{Scalar production}

First, we study the production of the heavier CP-even eigenstate, which we denote by $\mathcal{H}$ as above, at the LHC. Although the top coupling dominates the branching ratios parametrically, we find that the vector decay modes yield the strongest constraint. We show the limits in figure 7 and find again that most of the parameter space of the model is viable. However, the LHC search for $\mathcal{H}$ production in the $Z Z$ channel is in fact able to rule out the region stable up to the Planck scale for $m_{H}^{2}=0$.

The excluded cross sections at 95\% CL shown in the figure correspond to analyses using collisions at $13 \mathrm{TeV}$ in the center-of-mass energy to search for heavy resonances in $W W / W Z$ production in the decay channel $\ell \nu q q$ [29] (blue), $Z Z$ production in the decay channels $\ell^{+} \ell^{-} \ell^{+} \ell^{-}$and $\ell^{+} \ell^{-} \nu \bar{\nu}$ [30] (orange), $Z Z$ and $Z W$ production in the decay channels $\ell \ell q q$ and $\nu \nu q q$ [31] (red) and Higgs boson pairs decaying into $b b \tau \tau$ [32] (green). The decays of the light Higgs have been approximated by the SM prediction. The limits are shown in dashed lines.

An interesting point to note is that the $t \bar{t}$ decay channel dominates above threshold, cf. eq. (3.22). Resonant top-pair production is, however difficult to compute precisely due to large interference effects and QCD corrections [33-35]. The potential of the LHC experiments to observe this signal will rely on the ability to measure not only peak, but also other lineshape structures, such as peak-dip and pure dips. This search is also one of 


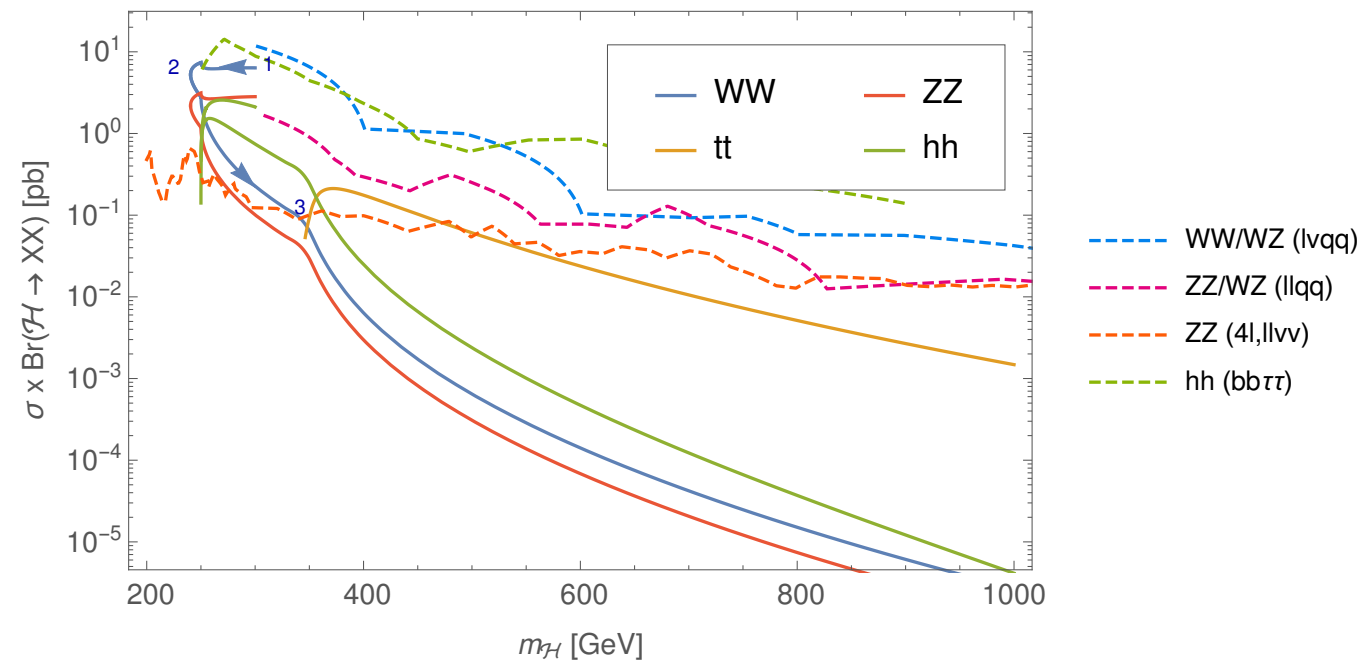

Figure 7. $\sigma \times \operatorname{Br}(\mathcal{H} \rightarrow X X)$ for the benchmark scenario with fixed $s_{\theta}=0.1$ and $m_{H}^{2}=0$. The arrow shows the growing $t_{\beta}$, and the points 1,2 , and 3 represent $t_{\beta}=1,1.7,3.6$, resp. The LHC limits are shown in dashed lines.

the most important programs of the LHC, since the top quark may well be related to new physics and has the least constrained interactions.

In the model an extra complication, which could in fact strengthen the signal, is the presence of near degenerate resonances: the three states, $\mathcal{H}, \pi^{0}$ and $\Pi_{5}$ have differences in mass of the order of few $\mathrm{GeV}$, as can be seen in the left panel of figure 1, and thus the peak of the top pair production cross-section is expected to be enhanced - although we note that the $\Pi_{5}$ decays into top quarks only via higher-scale physics [36]. Top quark pair production in the CP-conserving Type-II 2HDM with degenerate masses $m_{H}=m_{A}$ and including interference effect has been considered by the ATLAS collaboration at $\sqrt{s}=8 \mathrm{TeV}$ [37]. In the limit of small $s_{\theta}$, the model here is well approximated by this case. The exclusion limit at $95 \% \mathrm{CL}$ with $m_{H}=m_{A}=500 \mathrm{GeV}$ is $t_{\beta}=1.5$, and the exclusion limit decreases to approximately $t_{\beta}=0.9$ for $m_{H}=m_{A}=600 \mathrm{GeV}$. At these mass values, the $t_{\beta}$ values in the pCH models considered here are still significantly higher (cf. figure 1), but we expect improved results from Run II. For heavier masses, the analysis in ref. [37] is not optimal, and the boosted regime must be considered. In ref. [38] the LHC reach was studied for this kind of model through a reinterpretation of the top pair differential cross section measurement in both the boosted and resolved regimes. Although the near degenerate case was not considered, it seems to indicate that a very low systematic uncertainty must be achieved.

\subsection{3 $\quad B^{0}-\overline{B^{0}}$ mixing}

Models with two Higgs doublets, including bTC models [11, 13], are in general constrained by measurements of $B^{0}-\overline{B^{0}}$ mixing. Following [39], we calculate the contribution from the heavy pions to $B^{0}-\overline{B^{0}}$ mixing taking into account the next-to-leading order (NLO) 


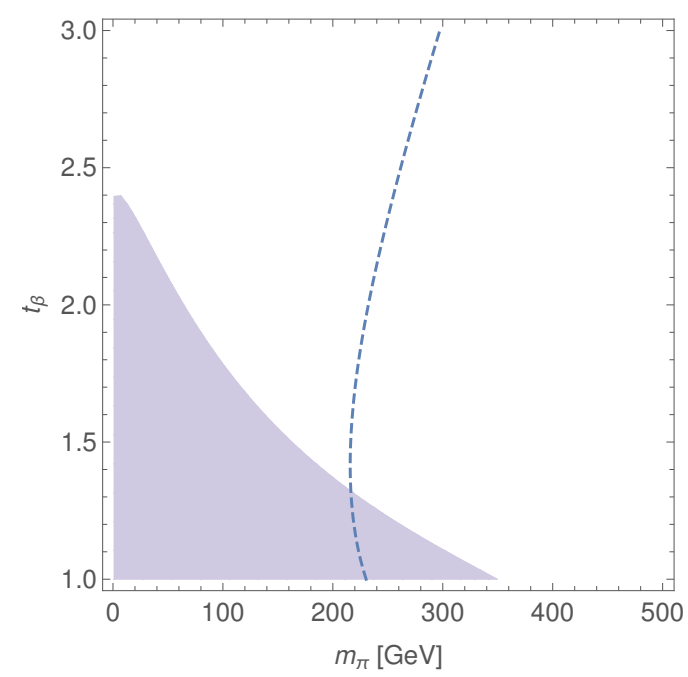

Figure 8. Excluded region by $B^{0}-\overline{B^{0}}$ mixing. The dashed curve shows the value of $m_{\pi}$ calculated for the benchmark scenario with $m_{H}^{2}=0$.

QCD corrections. The mass splitting can be written as

$$
\Delta m_{B}=\frac{G_{\mathrm{F}}^{2}}{6 \pi^{2}} m_{W}^{2}\left|V_{t d} V_{t b}^{*}\right|^{2} S\left(x_{W}, x_{\pi}\right) \eta\left(x_{W}, x_{\pi}\right) B_{B} f_{B}^{2} m_{B},
$$

where $x_{W, \pi} \equiv m_{t}^{2} / m_{W, \pi}^{2}$. The lengthy expressions for the functions $S$ and $\eta$ can be found in ref. [39] and we use the current QCD lattice estimate of the decay constant $f_{B} \sqrt{B_{B}}=$ $216 \pm 10 \mathrm{MeV}$ [40]. Using the experimental result $\Delta m_{B}=(3.3321 \pm 0.0013) \cdot 10^{-13} \mathrm{GeV}$ [41], we show the $2 \sigma$ exclusion region in the $\left(m_{\pi}, t_{\beta}\right)$ plane, along with the result for the pion mass for $m_{H}^{2}=0$ in figure 8 . The figure shows that the region with low $t_{\beta} \lesssim 1.5$ is in tension with also the $B^{0}-\overline{B^{0}}$ results.

\section{Model II: RG analysis and phenomenological constraints}

We now consider the analysis of our Model II where the SU(2) doublet of elementary scalars is extended to a full SU(4) multiplet $\Phi$. We refer to section 2.2 for a short review of the model and the effective potential.

\subsection{Vacuum and spectrum}

Following ref. [5], we proceed to minimize the effective potential given in eqs. (2.13)- (2.15). This yields equations of constraint for the SU(4) invariant Yukawa coupling, $y_{Q}$, the vev of the singlet scalar, $v_{S}$, and the $\mathrm{SU}(4)$-breaking mass parameter, $\delta m^{2}$ :

$$
y_{Q}=\frac{m_{\lambda}^{2} v}{8 \sqrt{2} \pi Z_{2} f^{3} s_{\theta}}, \quad v_{S}=\frac{\widetilde{C}_{g} Z_{2}^{2} f^{4} s_{\theta}^{2}-v^{2} m_{\lambda}^{2}}{t_{\theta} v m_{\lambda}^{2}}, \quad \delta m^{2}=\frac{\widetilde{C}_{g} Z_{2}^{2} f^{4} s_{\theta}^{2} m_{\lambda}^{2}}{v^{2} m_{\lambda}^{2}-\widetilde{C}_{g} Z_{2}^{2} f^{4} s_{\theta}^{2}},
$$

where $v \equiv\left\langle\sigma_{h}\right\rangle, v_{S} \equiv\left\langle S_{\mathrm{R}}\right\rangle, m_{\lambda}^{2} \equiv m_{\Phi}^{2}+\lambda_{\Phi}\left(v^{2}+v_{S}^{2}\right)$, and $\widetilde{C}_{g} \equiv C_{g}\left(3 g^{2}+g^{\prime 2}\right)$. 
The three CP-even neutral states $\sigma_{h}, \Pi_{4}, S_{\mathrm{R}}$ mix, but with the $\delta m^{2}$ perturbation in the scalar potential, we do not have simple analytical formulas for the mass mixings. We therefore solve numerically the rotation matrix, $R$, defined by

$$
\left(\begin{array}{c}
h_{1} \\
h_{2} \\
h_{3}
\end{array}\right) \equiv R\left(\begin{array}{c}
\sigma_{h} \\
\Pi_{4} \\
S_{\mathrm{R}}
\end{array}\right) .
$$

The lightest mass eigenstate, $h_{1}$, is identified with the observed Higgs boson with mass $m_{h_{1}}=125 \mathrm{GeV}$. The masses of the physical heavy pion triplet, $\pi^{ \pm, 0}$, orthogonal to those eaten by the $W$ and $Z$ bosons are given by

$$
\left(m_{\pi}^{ \pm, 0}\right)^{2}=\frac{8 \sqrt{2} \pi Z_{2} v_{\mathrm{w}}^{2} y_{Q}}{t_{\beta} s_{\theta}^{2}} .
$$

There are two additional CP-odd states in the spectrum: the mass eigenstates composed of $\Pi_{5}$ and $S_{\mathrm{I}}$. The spectrum for fixed $s_{\theta}=0.1$ is shown in figure 1 .

As shown in [5], it is also in this model possible to achieve the EWSB driven purely by the strong dynamics with $m_{\Phi}^{2}>0, \delta m^{2}>0$. In particular, in the viable parameter space able to produce the correct Higgs mass, the quartic coupling is generically enhanced with respect to the SM value. Furthermore, $\delta m^{2} \sim m_{\Phi}^{2} \sim f^{2}$, and thus for $s_{\theta} \lesssim 0.1$ the scalar mass parameters reach above $1 \mathrm{TeV}$.

\subsection{RG analysis}

The values of the new Yukawa couplings, $y_{Q}$, for successful vacuum solutions are very small, $y_{Q} \ll 1$ throughout the parameter space that we consider. We therefore neglect their contributions to the one-loop $\beta$-functions of the model given in appendix B.

The vacuum stability is improved with respect to Model I due to the enhanced quartic coupling, $\lambda_{\Phi}$, in most of the parameter space. However, the extended scalar sector now plays an important role because the SM-singlet elementary scalars, $S_{\mathrm{I}, \mathrm{R}}$, do not couple to SM fermions, and due to $y_{Q} \ll 1$ only very feebly to the techniquarks. Their quartic selfcouplings may therefore develop Landau poles, and in fact the bounds on parameter space from perturbativity of these couplings are more relevant than that coming from requiring vacuum stability. We calculate the scale, $\Lambda$, at which at least one of the couplings exceeds $4 \pi$, and plot the contours of $\Lambda=M_{\mathrm{Pl}}, 10^{15} \mathrm{GeV}, 10^{12} \mathrm{GeV}$ as dashed vertical lines in figure 9 along with the mass parameters $m_{\Phi}^{2}$ and $\delta m^{2}$. Below the cyan line on the left panel of figure $9, m_{\Phi}^{2}>0$, while $\delta m^{2}>0$ throughout, and the EWSB is completely induced by the composite sector. On the shaded gray region, the correct Higgs mass cannot be achieved. See ref. [5] for details.

One possibility to tame the running of the quartic couplings of the EW-singlet scalars $S_{\mathrm{I}, \mathrm{R}}$ is to couple these to right-handed neutrinos $\nu_{R}$ with $\lesssim \mathcal{O}(1)$ Yukawa couplings. This will drive the singlet quartics down, and result in higher perturbativity scale, as shown in ref. [42] in a generic framework with one singlet scalar and singlet fermion. Interestingly, in this case one family of leptons $e_{L}, \nu_{L}, e_{R}^{*}, \nu_{R}$ may be embedded into a full $\mathrm{SU}(4)$ multiplet 

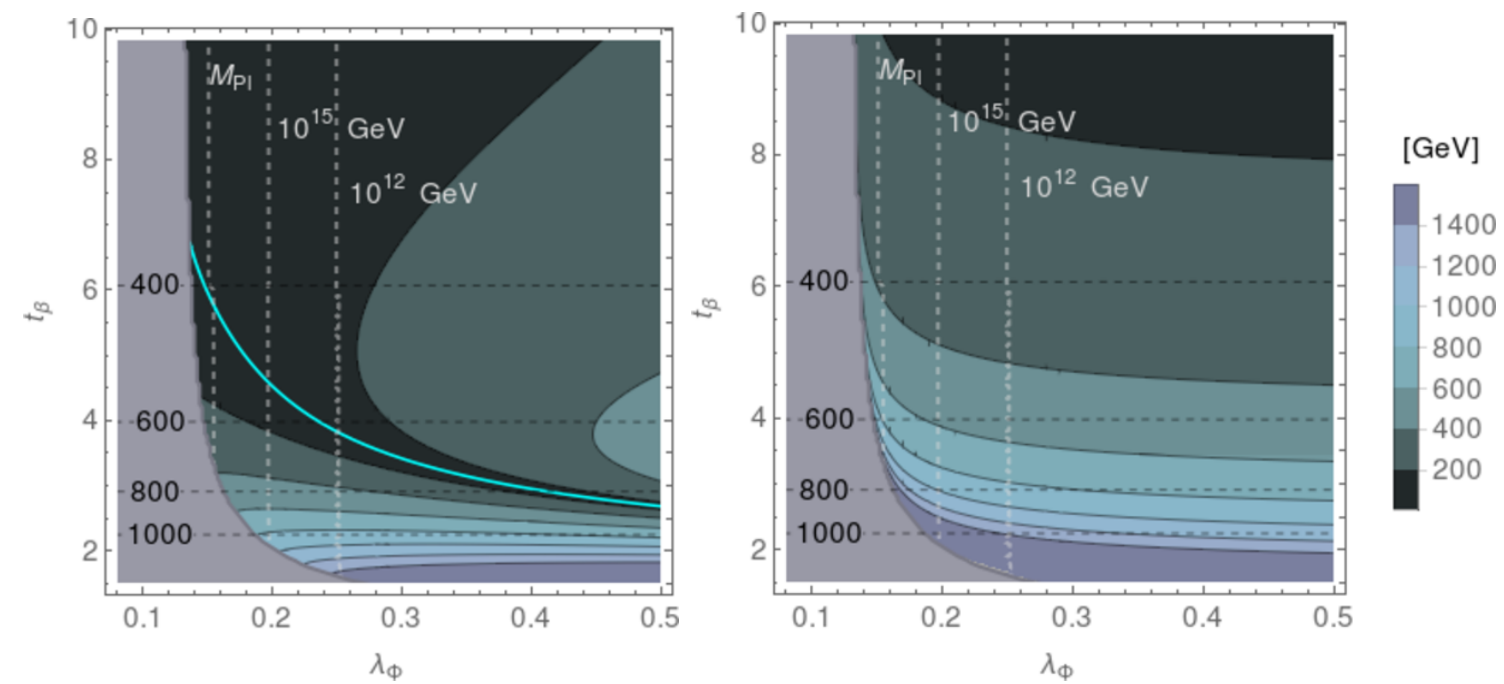

Figure 9. The values of mass parameters $\sqrt{\left|m_{\Phi}^{2}\right|}\left(\sqrt{\delta m^{2}}\right)$ are shown in left (right) panel when the Higgs mass is imposed for fixed $s_{\theta}=0.1$. Below the cyan line $m_{\Phi}^{2}>0$, while $\delta m^{2}>0$ throughout. On the gray region on the left, no solution to the Higgs-mass constraint is found. See ref. [5] for details. The dashed vertical lines show the parameter values where the maximum scale up to which the perturbativity of the coupligns can be attained is $10^{12} \mathrm{GeV}, 10^{15} \mathrm{GeV}$ or $M_{\mathrm{Pl}}$.

under the global symmetry analogously to the technifermions, as done in e.g. ref. [43]. The vev in the singlet direction would in this case give dynamical Majorana masses for the $\nu_{R}$ 's, while the vev in the doublet direction would yield a Dirac mass term. We leave the specifics of this construction for future work.

\subsection{Phenomenology}

The constraints from $B^{0}-\overline{B^{0}}$ mixing are not relevant in the parameter space considered in figure 9 because the scalar masses are now heavy in the range where $t_{\beta}$ is small and vice versa. The functions $S$ and $\eta$ in eq. (3.25) are therefore always small. The $\kappa$-coefficients for this model, defined as in eq. (3.20) but now with $i=1,2,3$, can be written in terms of the rotation matrix defined in eq. (4.2) as

$$
\kappa_{f, i}=\frac{R_{i 1}}{s_{\beta}}, \quad \kappa_{V, i}=R_{i 1} s_{\beta}+R_{i 2} c_{\beta} c_{\theta}, \quad i=1,2,3 .
$$

We refer to ref. [5] for an explicit illustration of the $\kappa_{f, 1}$-coefficient for fixed value of $s_{\theta}=0.1$. We conclude that for the most of the parameter space, the model is within the $2 \sigma$ region of two-parameter fit of $\kappa_{f, 1}, \kappa_{V, 1}$ on the combined CMS+ATLAS Run 1 data [28].

We present the collider limits relevant to the next to lightest neutral CP-even scalar, $h_{2}$, in figure 10. The constraint from searches for heavy neutral scalars in the $Z Z$ channel is reduced as compared to the $\mathrm{SU}(2)$-doublet-scalar case due to the additional mixing with the singlet $S_{\mathrm{R}}$. At face value, disregarding the fluctuations at small masses, the limit is about $225 \mathrm{GeV}$. In particular we note that the top coupling is significantly suppressed as compare to the Model I, and even at large mass the $t \bar{t}$ production is subdominant. 


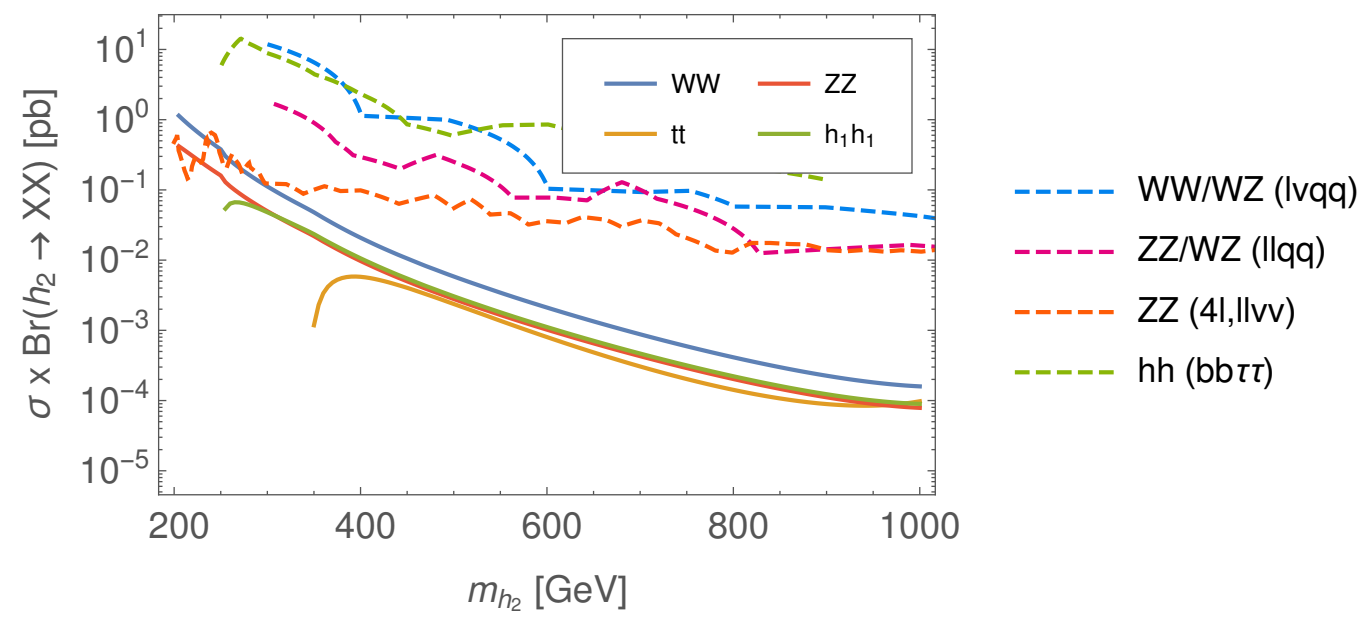

Figure 10. $\sigma \times \operatorname{Br}\left(h_{2} \rightarrow X X\right)$ in different channels with corresponding collider limits. We have fixed $\lambda_{\Phi}=0.15$ and $s_{\theta}=0.1$.

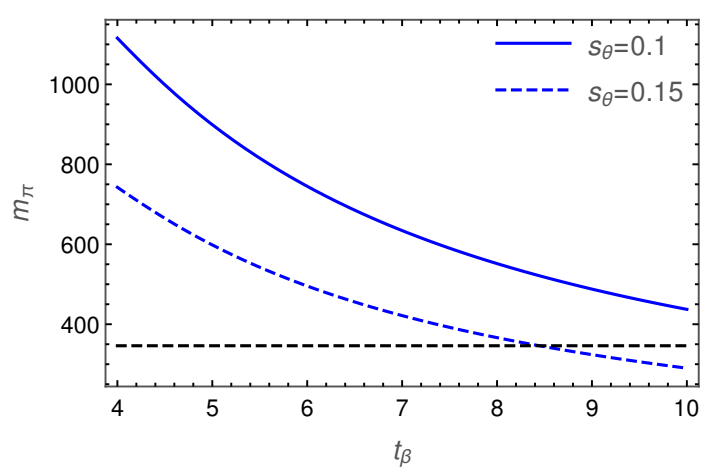

(a)

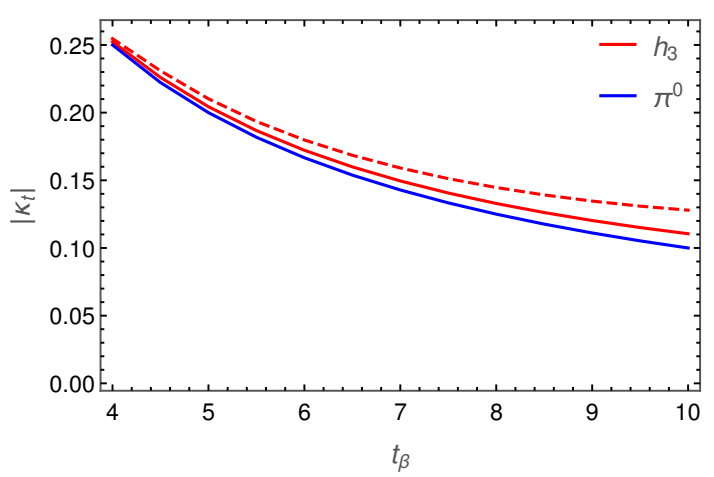

(b)

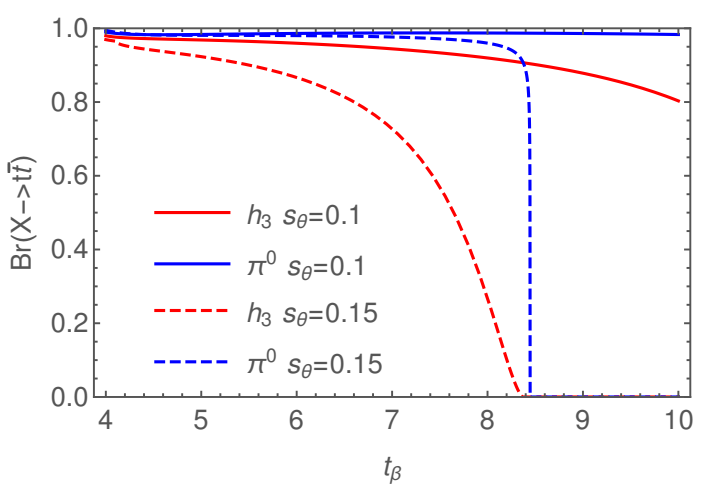

(c)

Figure 11. (a) Mass of the $\pi^{0}$. The dashed horizontal line shows the $t \bar{t}$ threshold. (b) Reduced coupling to top quarks. (c) Branching ratio of $h_{3}$ and $\pi^{0}$ to top-quark pair. In all panels $\lambda_{\Phi}=0.15$ and $s_{\theta}=0.1(0.15)$ in the solid (dashed) lines. 
On the other hand, the $\pi^{0}$ and $h_{3}$ states decay almost exclusively to $t \bar{t}$ for $s_{\theta} \lesssim 0.1$ as can be seen in the branching ratio to $t \bar{t}$ shown in figure 11(c) (off-shell decays were neglected in the figure). At larger values of $s_{\theta}$, the masses can get lower than the $t \bar{t}$ threshold, $m_{\pi^{0}}<2 m_{t}$, and other decay channels become important, cf. figure 11(a). In the case of $h_{3}$, the opening of other channels is more severe since it couples at tree level to weak bosons and to di-Higgs. For the $\pi^{0}$ state, the $Z h$ channel becomes dominant.

In the case where the top decay channel prevails, we can have a situation in which the LHC might be able to observe these states. To see this, we first note that these states are nearly degenerate in mass (see figure 1) and therefore they would be seen as a single summed peak at the LHC detectors. To access the magnitude of the peaks, we further note that the coupling of both states to top quarks are very similar, and thus also their total widths (in the case of top dominated) as can be seen in figure 11(b) (the variation with $\lambda_{\phi}$ is very small). Therefore, the production of both resonances can be effectively parametrized by the sum of the couplings. The magnitude of this sum could be within the expected reach of LHC, even for heavy masses, if the systematic uncertainty in $t \bar{t}$ can be mildly improved [38].

\section{Conclusions}

In this paper, we have studied the phenomenology of partially composite Higgs models based on the coset $\mathrm{SU}(4) / \mathrm{Sp}(4)$. In particular, we have considered the constraints on their parameter space from vacuum stability and perturbativity of the scalar self-couplings, together with constraints coming from Higgs coupling measurements, $B^{0}-\overline{B^{0}}$ mixing and LHC searches for new heavy scalars.

We considered two realisations of the elementary scalar sector: a minimal one with a single EW doublet coupled to the new fermions, and one with an SU(4) multiplet of scalars. In both models the new fermions feature vector-like current masses needed for the proper vacuum alignment; in the latter model they are dynamically generated by a vev of a EW-singlet component of the SU(4) scalar multiplet.

We have shown that in the minimal model there exists a region of allowed parameter values such that the vacuum remains stable and couplings perturbative up to the Planck scale. The EWSB is induced dynamically via the condensation in the strongly-interacting new fermion sector. However, it is only possible to push the value of the mass parameter of the elementary scalar towards the compositeness scale $\Lambda=4 \pi f$, and not beyond, at the expense of a lower vacuum stability scale. If we require stability of the vacuum up to the compositeness scale we find a bound on the mass parameter which is roughly $m_{H}^{2} \lesssim\left(0.1 s_{\theta}^{-1} \mathrm{TeV}\right)^{2}$. Conversely, the parameter region with vacuum stability up to the Planck scale lies near the classically scale-invariant region. However, we also show that LHC searches for new heavy CP-even scalars exclude this narrow stable window, while the constraints from a global fit to LHC Higgs coupling measurements and $B^{0}-\overline{B^{0}}$ mixing measurements are just beginning to probe this part of parameter space.

In summary, in most of the parameter space of the minimal model, the vacuum becomes unstable before the Planck scale due to the enhanced top-Yukawa coupling of the 
elementary scalar as compared to the SM Higgs. This issue can be alleviated, if the top quark acquires a small part of its mass, e.g. of the order of the $b$-quark mass, from some other source than the elementary scalar. One such source could be ETC-type four-fermion operators. However, large modifications in the bottom-Higgs coupling would then lead to tension with the measurements in the stability region.

The second model with a full SU(4) multiplet of scalars allows for dynamical generation of the vector-like masses of the new fermions, and the required explicit breaking of the SU(4) symmetry can be in the scalar potential as opposed to the Yukawa couplings in the minimal model. The quartic scalar couplings are in general enhanced compared to the SM removing the vacuum stability issue. However, due to the non-minimal scalar content, the singlet-scalar couplings instead develop Landau poles before the Planck scale in part of the parameter space. Again this limits the scalar mass parameters to the $\mathrm{TeV}$ region. One possibility to alleviate this problem is coupling the EW-singlet scalars to right-handed neutrinos. This model is also less constrained by current LHC searches and $B^{0}-\overline{B^{0}}$ mixing, but the top decay channel might provide an interesting possibility to observe the heavier scalar states of the model at the LHC in the future.

\section{Acknowledgments}

We thank A. Kagan for discussions. TA acknowledges partial funding from a Villum foundation grant when part of this article was being completed. MTF acknowledges partial funding from The Council For Independent Research, grant number DFF 6108-00623. The CP3-Origins centre is partially funded by the Danish National Research Foundation, grant number DNRF90. DBF acknowledges partial funding by the European Union as a part of the H2020 Marie Skłodowska-Curie Initial Training Network MCnetITN3 (722104).

\section{A RG equations: elementary doublet}

We consider a rather generic bTC framework featuring $N_{\mathrm{F}}$ new $\mathrm{SU}(2)_{\mathrm{L}}$-doublet fermionic fields, $Q_{\mathrm{L}}=\left(U_{\mathrm{L}}, D_{\mathrm{L}}\right)$, transforming under the representation $R_{\mathrm{F}}$ under the new strong gauge group, and coupling to the elementary Higgs doublet via Yukawa interactions. The one-loop evolution of the relevant couplings above the condensation scale, $\Lambda_{\mathrm{TC}}$, is given by

$$
\begin{aligned}
16 \pi^{2} \beta_{g_{\mathrm{c}}} & =-7 g_{\mathrm{c}}^{3} \\
16 \pi^{2} \beta_{g_{\mathrm{TC}}} & =-\left(\frac{11}{3} C_{2}(A)-\frac{8}{3} \cdot N_{\mathrm{F}} T\left(R_{\mathrm{F}}\right)\right) g_{\mathrm{TC}}^{3} \\
16 \pi^{2} \beta_{g_{\mathrm{L}}} & =-\left(\frac{19}{6}-\frac{2}{3} N_{\mathrm{F}} d\left(R_{\mathrm{F}}\right) T\left(R_{\mathrm{F}}\right)\right) g_{\mathrm{L}}^{3} \\
16 \pi^{2} \beta_{g_{Y}} & =\left(\frac{41}{6}+\frac{4}{3} N_{\mathrm{F}} d\left(R_{\mathrm{F}}\right)\left(4 Y\left(Q_{\mathrm{L}}\right)^{2}+\frac{1}{2}\right)\right) g_{Y}^{3} \\
16 \pi^{2} \beta_{y_{t}} & =\left(-8 g_{c}^{2}-\frac{9}{4} g_{\mathrm{L}}^{2}-\frac{17}{12} g_{Y}^{2}+\frac{9}{2} y_{t}^{2}+d\left(R_{\mathrm{F}}\right)\left(y_{U}^{2}+y_{D}^{2}\right)\right) y_{t}, \\
16 \pi^{2} \beta_{y_{U}} & =\left(-6 C_{2}\left(R_{\mathrm{F}}\right) g_{\mathrm{TC}}^{2}-\frac{9}{4} g_{\mathrm{L}}^{2}-\frac{17}{12} g_{Y}^{2}+3 y_{t}^{2}+\left(d\left(R_{\mathrm{F}}\right)+\frac{3}{2}\right) y_{U}^{2}+d\left(R_{\mathrm{F}}\right) y_{D}^{2}\right) y_{U},
\end{aligned}
$$




$$
\begin{aligned}
16 \pi^{2} \beta_{y_{D}}= & \left(-6 C_{2}\left(R_{\mathrm{F}}\right) g_{\mathrm{TC}}^{2}-\frac{9}{4} g_{\mathrm{L}}^{2}-\frac{5}{12} g_{Y}^{2}+3 y_{t}^{2}+\left(d\left(R_{\mathrm{F}}\right)+\frac{3}{2}\right) y_{D}^{2}+d\left(R_{\mathrm{F}}\right) y_{U}^{2}\right) y_{D} \\
16 \pi^{2} \beta_{\lambda}= & 24 \lambda^{2}+\lambda\left(-3\left(3 g_{\mathrm{L}}^{2}+g_{Y}^{2}\right)+12 y_{t}^{2}+4 d\left(R_{\mathrm{F}}\right)\left(y_{U}^{2}+y_{D}^{2}\right)\right) \\
& \left.+\frac{3}{8}\left(2 g_{\mathrm{L}}^{4}+\left(g_{\mathrm{L}}^{2}+g_{Y}^{2}\right)^{2}\right)\right)-6 y_{t}^{4}-2 d\left(R_{\mathrm{F}}\right)\left(y_{U}^{4}+y_{D}^{4}\right)
\end{aligned}
$$

where

$$
\beta_{g} \equiv \frac{\mathrm{d} g}{\mathrm{~d} \log \mu},
$$

$T\left(R_{\mathrm{F}}\right)$ and $d\left(R_{\mathrm{F}}\right)$ are the index and dimension of the representation $R_{\mathrm{F}}$, and $C_{2}(A)$ is the quadratic Casimir of the adjoint representation.

\section{B RG equations: SU(4) multiplet of elementary scalars}

Here we write down the RG equations for specific bTC framework based on $\mathrm{SU}(4) / \mathrm{Sp}(4)$ coset described in the main text. The new $\mathrm{SU}(2)_{\mathrm{L}}$-doublet fermionic fields, $Q_{\mathrm{L}}=\left(U_{\mathrm{L}}, D_{\mathrm{L}}\right)$, transform in the fundamental representation of the new strong gauge group, and couple to the multiplet of elementary scalars transforming in the two-index antisymmetric representation of SU(4) via Yukawa interactions. From the RG point of view, the scalar sector corresponds to an EW doublet and two singlets, and the SU(4) invariance of the potential can be achieved only for one renormalisation scale $\mu_{0}$. The quartic scalar potential can be written as

$$
V^{(4)}=\lambda_{H}\left(H^{\dagger} H\right)^{2}+\frac{\lambda_{S_{R}}}{4} S_{R}^{4}+\frac{\lambda_{S_{I}}}{4} S_{I}^{4}+\lambda_{H S_{R}}\left(H^{\dagger} H\right) S_{R}^{2}+\lambda_{H S_{I}}\left(H^{\dagger} H\right) S_{I}^{2}+\frac{\lambda_{S_{R} S_{I}}}{2} S_{R}^{2} S_{I}^{2},
$$

with $\mathrm{SU}(4)$-symmetric boundary values $\lambda_{H}\left(\mu_{0}\right)=\lambda_{S_{R}}\left(\mu_{0}\right)=\lambda_{S_{I}}\left(\mu_{0}\right)=\lambda_{H S_{R}}\left(\mu_{0}\right)=$ $\lambda_{H S_{I}}\left(\mu_{0}\right)=\lambda_{S_{R} S_{I}}\left(\mu_{0}\right)=\lambda_{\Phi}$

The one-loop evolution of the relevant couplings above the condensation scale, $\Lambda_{\mathrm{TC}}$, is given by (we ignore the new Yukawa couplings, since for the viable parameter points able to give the correct Higgs mass, $y_{Q} \lesssim 0.1$ throughout the parameter space we consider, and are thus their contribution to the $\beta$-functions is completely subdominant)

$$
\begin{aligned}
16 \pi^{2} \beta_{g_{\mathrm{TC}}}= & -6 g_{\mathrm{TC}}^{3}, \quad 16 \pi^{2} \beta_{g_{\mathrm{L}}}=-\frac{5}{2} g_{\mathrm{L}}^{3}, \quad 16 \pi^{2} \beta_{g_{Y}}=\frac{15}{2} g_{Y}^{3}, \\
16 \pi^{2} \beta_{y_{t}}= & \left(-8 g_{c}^{2}-\frac{9}{4} g_{\mathrm{L}}^{2}-\frac{17}{12} g_{Y}^{2}+\frac{9}{2} y_{t}^{2}\right) y_{t}, \\
16 \pi^{2} \beta_{\lambda_{H}}= & 24 \lambda_{H}^{2}+2\left(\lambda_{H S_{R}}^{2}+\lambda_{H S_{I}}^{2}\right)+\lambda_{H}\left(-3\left(3 g_{\mathrm{L}}^{2}+g_{Y}^{2}\right)+12 y_{t}^{2}\right) \\
& \left.+\frac{3}{8}\left(2 g_{\mathrm{L}}^{4}+\left(g_{\mathrm{L}}^{2}+g_{Y}^{2}\right)^{2}\right)\right)-6 y_{t}^{4}, \\
16 \pi^{2} \beta_{\lambda_{H S_{R}}}= & 8 \lambda_{H S_{R}}^{2}+6\left(2 \lambda_{H}+\lambda_{S_{R}}\right) \lambda_{H S_{R}}+2 \lambda_{H S_{I}} \lambda_{S_{R} S_{I}} \\
& +\lambda_{H S_{R}}\left(-\frac{3}{2}\left(3 g_{\mathrm{L}}^{2}+g_{Y}^{2}\right)+6 y_{t}^{2}\right), \\
16 \pi^{2} \beta_{\lambda_{H S_{I}}=} & 8 \lambda_{H S_{I}}^{2}+6\left(2 \lambda_{H}+\lambda_{S_{I}}\right) \lambda_{H S_{I}}+2 \lambda_{H S_{R}} \lambda_{S_{R} S_{I}} \\
& +\lambda_{H S_{I}}\left(-\frac{3}{2}\left(3 g_{\mathrm{L}}^{2}+g_{Y}^{2}\right)+6 y_{t}^{2}\right),
\end{aligned}
$$




$$
\begin{aligned}
16 \pi^{2} \beta_{\lambda_{S_{R}}} & =18 \lambda_{S_{R}}^{2}+8 \lambda_{H S_{R}}^{2}+2 \lambda_{S_{R} S_{I}}^{2}, \\
16 \pi^{2} \beta_{\lambda_{S_{I}}} & =18 \lambda_{S_{I}}^{2}+8 \lambda_{H S_{I}}^{2}+2 \lambda_{S_{R} S_{I}}^{2}, \\
16 \pi^{2} \beta_{\lambda_{S_{R} S_{I}}} & =8 \lambda_{S_{R} S_{I}}^{2}+6 \lambda_{S_{R} S_{I}}\left(\lambda_{S_{R}}+\lambda_{S_{I}}\right)+8 \lambda_{H S_{R}} \lambda_{H S_{I}} .
\end{aligned}
$$

Open Access. This article is distributed under the terms of the Creative Commons Attribution License (CC-BY 4.0), which permits any use, distribution and reproduction in any medium, provided the original author(s) and source are credited.

\section{References}

[1] D.B. Kaplan and H. Georgi, $\mathrm{SU}(2) \times \mathrm{U}(1)$ breaking by vacuum misalignment, Phys. Lett. 136B (1984) 183 [INSPIRE].

[2] J. Galloway, A.L. Kagan and A. Martin, A UV complete partially composite-PNGB Higgs, Phys. Rev. D 95 (2017) 035038 [arXiv: 1609.05883] [INSPIRE].

[3] A. Agugliaro et al., UV complete composite Higgs models, Phys. Rev. D 95 (2017) 035019 [arXiv: 1609.07122] [INSPIRE].

[4] T. Alanne, M.T. Frandsen and D. Buarque Franzosi, Testing a dynamical origin of Standard Model fermion masses, Phys. Rev. D 94 (2016) 071703 [arXiv:1607.01440] [INSPIRE].

[5] T. Alanne, D. Buarque Franzosi and M.T. Frandsen, A partially composite Goldstone Higgs, Phys. Rev. D 96 (2017) 095012 [arXiv:1709.10473] [InSPIRE].

[6] G. 't Hooft, Naturalness, chiral symmetry, and spontaneous chiral symmetry breaking, NATO Sci. Ser. B $\mathbf{5 9}$ (1980) 135.

[7] D.B. Kaplan, Flavor at SSC energies: a New mechanism for dynamically generated fermion masses, Nucl. Phys. B 365 (1991) 259 [InSPIRE].

[8] E. Eichten and K.D. Lane, Dynamical breaking of weak interaction symmetries, Phys. Lett. 90B (1980) 125 [INSPIRE].

[9] S. Dimopoulos and L. Susskind, Mass without scalars, Nucl. Phys. B 155 (1979) 237 [INSPIRE].

[10] D.B. Kaplan, H. Georgi and S. Dimopoulos, Composite Higgs scalars, Phys. Lett. 136B (1984) 187 [INSPIRE].

[11] E.H. Simmons, Phenomenology of a technicolor model with heavy scalar doublet, Nucl. Phys. B 312 (1989) 253 [INSPIRE].

[12] A. Kagan and S. Samuel, The family mass hierarchy problem in bosonic technicolor, Phys. Lett. B 252 (1990) 605 [INSPIRE].

[13] C.D. Carone, Technicolor with a 125 GeV Higgs boson, Phys. Rev. D 86 (2012) 055011 [arXiv: 1206.4324] [INSPIRE].

[14] G. Ferretti and D. Karateev, Fermionic UV completions of composite Higgs models, JHEP 03 (2014) 077 [arXiv: 1312.5330] [INSPIRE].

[15] R. Lewis, C. Pica and F. Sannino, Light asymmetric dark matter on the lattice: SU(2) technicolor with two fundamental flavors, Phys. Rev. D 85 (2012) 014504 [arXiv:1109.3513] [INSPIRE]. 
[16] M.A. Luty and T. Okui, Conformal technicolor, JHEP 09 (2006) 070 [hep-ph/0409274] [INSPIRE].

[17] J. Galloway, J.A. Evans, M.A. Luty and R.A. Tacchi, Minimal conformal technicolor and precision electroweak tests, JHEP 10 (2010) 086 [arXiv:1001.1361] [INSPIRE].

[18] G. Cacciapaglia and F. Sannino, Fundamental composite (Goldstone) Higgs dynamics, JHEP 04 (2014) 111 [arXiv: 1402.0233] [INSPIRE].

[19] D. Buarque Franzosi, G. Cacciapaglia, H. Cai, A. Deandrea and M. Frandsen, Vector and axial-vector resonances in composite models of the Higgs boson, JHEP 11 (2016) 076 [arXiv: 1605.01363] [INSPIRE].

[20] S. Weinberg, Implications of dynamical symmetry breaking, Phys. Rev. D 13 (1976) 974 [INSPIRE].

[21] L. Susskind, Dynamics of spontaneous symmetry breaking in the Weinberg-Salam theory, Phys. Rev. D 20 (1979) 2619 [inSPIRE].

[22] R. Arthur, V. Drach, M. Hansen, A. Hietanen, C. Pica and F. Sannino, SU(2) gauge theory with two fundamental flavors: a minimal template for model building, Phys. Rev. D 94 (2016) 094507 [arXiv: 1602.06559] [INSPIRE].

[23] M.E. Peskin, The alignment of the vacuum in theories of technicolor, Nucl. Phys. B 175 (1980) 197 [INSPIRE].

[24] J. Preskill, Subgroup alignment in hypercolor theories, Nucl. Phys. B 177 (1981) 21 [INSPIRE].

[25] G. Degrassi et al., Higgs mass and vacuum stability in the Standard Model at NNLO, JHEP 08 (2012) 098 [arXiv: 1205.6497] [INSPIRE].

[26] W.J. Marciano, Exotic new quarks and dynamical symmetry breaking, Phys. Rev. D 21 (1980) 2425 [INSPIRE].

[27] G. Cacciapaglia and F. Sannino, An ultraviolet chiral theory of the top for the fundamental composite (goldstone) Higgs, Phys. Lett. B 755 (2016) 328 [arXiv: 1508.00016] [InSPIRE].

[28] ATLAS and CMS collaborations, Measurements of the Higgs boson production and decay rates and constraints on its couplings from a combined ATLAS and CMS analysis of the LHC pp collision data at $\sqrt{s}=7$ and $8 \mathrm{TeV}$, JHEP 08 (2016) 045 [arXiv:1606.02266] [INSPIRE].

[29] ATLAS collaboration, Search for $W W / W Z$ resonance production in $\ell \nu q q$ final states in $p p$ collisions at $\sqrt{s}=13 \mathrm{TeV}$ with the ATLAS detector, arXiv:1710.07235.

[30] ATLAS collaboration, Search for heavy $Z Z$ resonances in the $\ell^{+} \ell^{-} \ell^{+} \ell^{-}$and $\ell^{+} \ell^{-} \nu \bar{\nu}$ final states using proton-proton collisions at $\sqrt{s}=13 \mathrm{TeV}$ with the ATLAS detector, arXiv: 1712.06386.

[31] ATLAS collaboration, Searches for heavy $Z Z$ and $Z W$ resonances in the $\ell \ell q q$ and $\nu \nu q q$ final states in pp collisions at $\sqrt{s}=13 \mathrm{TeV}$ with the ATLAS detector, arXiv:1708.09638 [INSPIRE].

[32] CMS collaboration, Search for Higgs boson pair production in events with two bottom quarks and two $\tau$ leptons in proton-proton collisions at $\sqrt{s}=13 \mathrm{TeV}$, arXiv:1707.02909 [INSPIRE].

[33] W. Bernreuther et al., Production of heavy Higgs bosons and decay into top quarks at the LHC, Phys. Rev. D 93 (2016) 034032 [arXiv:1511.05584] [InSPIRE]. 
[34] B. Hespel, F. Maltoni and E. Vryonidou, Signal background interference effects in heavy scalar production and decay to a top-anti-top pair, JHEP 10 (2016) 016 [arXiv:1606.04149] [INSPIRE].

[35] D. Buarque Franzosi, E. Vryonidou and C. Zhang, Scalar production and decay to top quarks including interference effects at NLO in QCD in an EFT approach, JHEP 10 (2017) 096 [arXiv:1707.06760] [INSPIRE].

[36] A. Arbey et al., Fundamental composite electroweak dynamics: status at the LHC, Phys. Rev. D 95 (2017) 015028 [arXiv: 1502.04718] [INSPIRE].

[37] ATLAS collaboration, Search for heavy higgs bosons $A / H$ decaying to a top quark pair in $p p$ collisions at $\sqrt{s}=8 \mathrm{TeV}$ with the ATLAS detector, Phys. Rev. Lett. 119 (2017) 191803 [arXiv: 1707.06025] [INSPIRE].

[38] D. Buarque Franzosi, F. Fabbri and S. Schumann, Constraining scalar resonances with top-quark pair production at the LHC, arXiv:1711.00102 [INSPIRE].

[39] J. Urban, F. Krauss, U. Jentschura and G. Soff, Next-to-leading order QCD corrections for the $B^{0}-\bar{B}^{0}$ mixing with an extended Higgs sector, Nucl. Phys. B 523 (1998) 40 [hep-ph/9710245] [inSPIRE].

[40] ETM collaboration, N. Carrasco et al., B-physics from $N_{f}=2$ tmQCD: the Standard Model and beyond, JHEP 03 (2014) 016 [arXiv: 1308.1851] [INSPIRE].

[41] Particle Data Group collaboration, C. Patrignani et al., Review of particle physics, Chin. Phys. C 40 (2016) 100001.

[42] T. Alanne, K. Tuominen and V. Vaskonen, Strong phase transition, dark matter and vacuum stability from simple hidden sectors, Nucl. Phys. B 889 (2014) 692 [arXiv:1407.0688] [INSPIRE].

[43] R. Foadi, M.T. Frandsen, T.A. Ryttov and F. Sannino, Minimal walking technicolor: set up for collider physics, Phys. Rev. D 76 (2007) 055005 [arXiv:0706.1696] [INSPIRE]. 